\title{
Classification of Stokes Graphs of Second Order Fuchsian Differential Equations of Genus Two
}

\author{
By \\ Takashi Aokı* and Takayuki IızUKA**
}

\begin{abstract}
Stokes curves of second order Fuchsian differential equations on the Riemann sphere with a large parameter form sphere graphs, which are called Stokes graphs. Topological classification of Stokes graphs are given for the case where equations have five regular singular points. It is proved that there are exactly 25 degree sequences of sphere triangulations associated with Stokes graphs under suitable generic conditions.
\end{abstract}

\section{$\S 1 . \quad$ Introduction}

Let $F(x)$ and $G(x)$ be polynomials of $x$ with complex coefficients of degree $2 g+2$ and $g+2$, respectively. Here $g$ is a non-negative integer. We set

$$
Q(x)=\frac{F(x)}{G(x)^{2}}
$$

and consider the following differential equation with a large parameter $\eta$ :

$$
\left(-\frac{d^{2}}{d x^{2}}+\eta^{2} Q(x)\right) \psi=0 .
$$

Let $a_{j}(j=0,1, \ldots, 2 g+1)$ denote the zeros of $F$ and let $b_{j}(j=0,1, \ldots, g+1)$ denote the zeros of $G$. We assume that $a_{j}$ 's and $b_{j}$ 's are mutually distinct. Then (1.1) is a second order Fuchsian differential equation with regular singularities

Communicated by T. Kawai. Received April 17, 2006.

2000 Mathematics Subject Classification(s): 34M40, 34M60, 05C10.

The first author is supported in part by JSPS Grant-in-Aid No. 15540190.

*Department of Mathematics, Kinki University, Higashi-Osaka 577-8502, Japan.

** Department of Mathematics, Kinki University, Higashi-Osaka 577-8502, Japan.

(c) 2007 Research Institute for Mathematical Sciences, Kyoto University. All rights reserved. 
at $x=b_{j}(j=0,1, \ldots, g+1)$ and $x=\infty$. We call $a_{j}$ a turning point of (1.1). A Stokes curve is, by definition, an integral curve of the direction field

$$
\operatorname{Im} \sqrt{Q(x)} d x=0
$$

starting at a turning point. The set of all Stokes curves, $\left\{a_{j}\right\}$ and $\left\{b_{j}\right\}$ form a graph with vertex 2 -coloring on $\mathbb{P}_{\mathbb{C}}^{1} \approx S^{2}$. This graph is called the Stokes graph of (1.1). The integer $g$ is said to be the genus of (1.1) or of the graph. Here we note that $g$ is the genus of the Riemann surface defined by $y^{2}=Q(x)$. Stokes graphs play a rôle in the global analysis of (1.1) by means of the exact(complex) WKB analysis such as computations of Stokes coefficients (cf. [6]) or of monodromy matrices (cf. [4]). Topological classification of Stokes graphs are given in [5] (see also [4]) for $g=0,1$ under some suitable generic conditions. That is, there are two types of Stokes graphs in the case of $g=0$ and six types for the case of $g=1$. The aim of this article is to give all the topological types of Stokes graphs in the case of $g=2$. We find 25 types in our case. Our method is based on the observations given in [5], [4]. The classification of the Stokes graphs is reduced to that of triangulations of $S^{2}$ of a special kind, which we call specific triangulations. Thus we shall classify such triangulations in the case of $g=2$. However, some combinatorial complexities appear in our case. To construct all possible configurations of specific triangulations, we develop two procedures for such triangulations, namely, reduction and blow up. One of our main results (Theorem 2.3) is announced in [3]. In Appendix we give examples of potentials $Q$ which realize 25 types of Stokes graphs of our classification.

\section{§2. Stokes Graphs and Specific Triangulations of the Riemann Sphere}

\section{$\S 2.1$. Triangles}

Let $\Delta$ denote the open triangle in $\mathbb{R}^{2}$ defined by

$$
\Delta=\{(x, y) \mid x+y<1, x>0, y>0\} .
$$

The sides of $\Delta$ are denoted by $s_{j}(j=1,2,3)$ :

$$
\begin{aligned}
& s_{1}=\{(z, 0) \mid 0<z<1\}, \\
& s_{2}=\{(1-z, z) \mid 0<z<1\}, \\
& s_{3}=\{(0,1-z) \mid 0<z<1\} .
\end{aligned}
$$

We denote by $t_{j}(j=1,2,3)$ the vertices of $\Delta$ :

$$
t_{1}=(0,1), t_{2}=(0,0), t_{3}=(1,0) .
$$


The starting point and the endpoint of $s_{j}$ are the boundary points of $s_{j}$ corresponding to $z=0$ and to $z=1$ in the expressions given by (2.2)-(2.4), respectively. For example, $t_{1}$ is the starting point of $s_{3}$ and, at the same time, it is the endpoint of $s_{2}$. We set

$$
\widetilde{\Delta}=\Delta \cup s_{1} \cup s_{2} \cup s_{3}
$$

and

$$
\bar{\Delta}=\tilde{\Delta} \cup\left\{t_{1}\right\} \cup\left\{t_{2}\right\} \cup\left\{t_{3}\right\}
$$

A triangle on the Riemann sphere $\mathbb{P}_{\mathbb{C}}^{1}$ means a continuous mapping

$$
f: \bar{\Delta} \longrightarrow \mathbb{P}_{\mathbb{C}}^{1}
$$

so that the restrictions of $f$ to $\Delta$ and to each $s_{j}$ are injective. Sometimes the image of $\bar{\Delta}$ (or $\tilde{\Delta}, \Delta$ ) by $f$ is also called a triangle in $\mathbb{P}_{\mathbb{C}}^{1}$. Similarly, the images of sides and vertices by $f$ are called sides and vertices of $f(\bar{\Delta})$, respectively. Sides are called edges if they are considered to be elements of graphs on $\mathbb{P}_{\mathbb{C}}^{1}$.

\section{$\S 2.2$. Specific triangulations}

Let $g$ be a non-negative integer. A specific triangulation of $\mathbb{P}_{\mathbb{C}}^{1}$ of genus $g$ is, by definition, a set of $2 g+2$ continuous mappings

$$
f_{i}: \bar{\Delta} \longrightarrow \mathbb{P}_{\mathbb{C}}^{1} \quad(i=1,2, \ldots, 2 g+2)
$$

satisfying the following conditions:

1. The union of the sets $f_{i}(\bar{\Delta})(i=1,2, \ldots, 2 g+2)$ coincides with $\mathbb{P}_{\mathbb{C}}^{1}$.

2. If $i \neq j$, the intersection of $f_{i}(\Delta)$ and $f_{j}(\Delta)$ is empty.

3. If the intersection of $f_{i}(\tilde{\Delta})$ and $f_{j}(\tilde{\Delta})$ is not empty for some $i \neq j$, then there exist $k, l(1 \leq k, l \leq 3)$ for which $f_{i}\left(s_{k}\right)=f_{j}\left(s_{l}\right)$ holds.

4. If the intersection of $f_{j}\left(s_{k}\right)$ and $f_{j}\left(s_{l}\right)$ is not empty for some $k, l(1 \leq$ $k, l \leq 3)$, then $f_{j}\left(s_{k}\right)=f_{j}\left(s_{l}\right)$ holds.

5. The set of all vertices $V=\left\{f_{j}\left(t_{k}\right) \mid 1 \leq j \leq 2 g+2,1 \leq k \leq 3\right\}$ contains exactly $g+3$ points.

6. The set $\bigcup_{j=1}^{2 g+2} \bigcup_{k=1}^{3} f_{j}\left(s_{k}\right)$ has $3 g+3$ connected components. 
For a given specific triangulation $\left\{f_{i}\right\}$ of $\mathbb{P}_{\mathbb{C}}^{1}$ of genus $g$, we can make a sphere graph $\mathcal{T}=(V, E, F)$. Here $E$ denotes the set of all connected components $2 g+2 \quad 3$

of $\bigcup_{j=1}^{2 g} \bigcup_{k=1}^{3} f_{j}\left(s_{k}\right)$ and each connected component is regarded as an edge of $\mathcal{T}$ and here $F$ denotes the set $\left\{f_{i}(\tilde{\Delta})\right\}$ of faces. We also call $\mathcal{T}$ a specific triangulation of $\mathbb{P}_{\mathbb{C}}^{1}$ of genus $g$. Two specific triangulations $\mathcal{T}$ and $\mathcal{T}^{\prime}$ are said to be equivalent if $\mathcal{T}$ and $\mathcal{T}^{\prime}$ are isomorphic as sphere graphs. An equivalence class of specific triangulations is called an abstract specific triangulation. Note that we sometimes identify sides of triangles with edges of the triangulation if there is no confusion.

\section{§2.3. Stokes graphs and specific triangulations}

We briefly review some basic properties of Stokes graphs of (1.1) after [4]. For every turning point $a_{i}$, there are three Stokes curves $l_{i, j}(j=1,2,3)$ that emanate from $a_{i}$. Each Stokes curve $l_{i, j}$ terminates at some regular singular point $b_{k}$ under suitable generic conditions for (1.1) (cf. [4], Chapter 3). Let $A$, $B$ and $L$ denote the sets of all turning points, of all regular singular points and of all Stokes curves, respectively. The triplet $\mathcal{S}=(A, B, L)$ can be regarded as a sphere (multi-)graph with vertex 2-coloring. That is, the elements of $A$ and $B$ are considered to be vertices of color $A$ and of color $B$, respectively and the Stokes curves are edges of the graph. We call this graph the Stokes graph of (1.1). The faces of the graph are quadrangles and the number of faces is $3 g+3$, where $g$ is the genus of (1.1). Two Stokes graphs $\mathcal{S}$ and $\mathcal{S}^{\prime}$ are said to be equivalent if they are isomorphic as sphere graphs with vertex 2-coloring. An equivalence class of Stokes graphs can be regarded as an abstract Stokes graph ([4], Definition 3.9).

For a given abstract Stokes graph $\mathcal{S}=(A, B, L)$, we denote by $M$ the set of all faces of $\mathcal{S}$. We consider a new sphere graph $\mathcal{G}^{*}=(B, M, A)$. That is, the vertices, the edges and the faces of $\mathcal{G}^{*}$ are the vertices $b_{j}$ of color $B$, the faces $M_{j}$ and the vertices $a_{j}$ of color $A$ of $\mathcal{S}$, respectively. We consider $b_{j}$ is incident with an edge $M_{k}$ if $b_{j}$ is contained in the topological boundary of $M_{k}$. Similarly, an edge $M_{j}$ is considered to be incident with a face $a_{k}$ if $a_{k}$ is contained in the topological boundary of $M_{j}$. Then $\mathcal{G}^{*}$ can be regarded as an abstract specific triangulation of the sphere. We call $\mathcal{G}^{*}$ the specific triangulation associated with $\mathcal{S}$. Thus graph theoretic classification of abstract Stokes graphs is reduced to that of abstract specific triangulations.

Let $\mathcal{T}=(V, E, F)$ be a specific triangulation of genus $g$ of the sphere. Let $m_{j}$ be the number of edges incident with vertex $v_{j}$, where a loop is counted 
by two. We call $m_{j}$ the degree of $v_{j}$. We arrange the order of the sequence $\left\{m_{j}\right\}$ monotonically decreasingly and denote it by $d_{1} \geq d_{2} \geq \cdots \geq d_{g+3}$. We set $\boldsymbol{d}=\left(d_{1}, d_{2}, \ldots, d_{g+3}\right)$ and call it the index of $\mathcal{T}$. Of course the notion of index can be defined also for abstract specific triangulations. Note that $\sum_{k=1}^{g+3} d_{k}=6 g+6$ holds.

Definition 2.1. A multi-index $\boldsymbol{d}=\left(d_{1}, d_{2}, \ldots, d_{g+3}\right)\left(d_{i} \in \boldsymbol{N}\right)$ is called an admissible index of genus $g$ if there exists a specific triangulation $\mathcal{T}$ of genus $g$ of the sphere whose index is equal to $\boldsymbol{d}$.

Let $\mathcal{S}=(A, B, L)$ be a Stokes graph of (1.1) of genus $g$. Let $m_{j}^{\prime}$ denote the number of Stokes curves which terminate at $b_{j}$. Let $\mathcal{G}^{*}$ be the specific triangulation associated with $\mathcal{S}$. Note that $m_{j}^{\prime}$ coincides with the number of connected components of the intersection of a sufficiently small disk with the center at $b_{j}$ and the union of faces of $\mathcal{S}$ whose boundaries contain $b_{j}$. Hence $m_{j}^{\prime}$ is equal to the degree of $b_{j}$. Thus the index of the associated graph $\mathcal{G}^{*}$ is an invariant of $\mathcal{S}$ as well and we may call it the index of $\mathcal{S}$.

\section{§2.4. Classification of specific triangulations of genus two}

Let us consider the case where $g=2$. As we saw in the preceding subsection, the index $\boldsymbol{d}=\left(d_{1}, d_{2}, \ldots, d_{5}\right)$ of a given specific triangulation of genus two satisfies

$$
\sum_{k=1}^{5} d_{k}=18 .
$$

There are 57 solutions $\boldsymbol{d}=\left(d_{1}, d_{2}, \ldots, d_{5}\right)$ of (2.5) satisfying $d_{1} \geq d_{2} \geq \cdots \geq d_{5}$, $d_{k} \in \boldsymbol{N}$. Not all but 25 solutions of them are admissible. That is, we have

Theorem 2.1. Let $\mathcal{T}$ be a specific triangulation of genus two of the sphere. Then the index $\boldsymbol{d}$ of $\mathcal{T}$ coincides with one of the following 25 multiindices:

$$
\begin{aligned}
& (4,4,4,3,3), \quad(5,5,3,3,2), \quad(6,4,4,2,2), \quad(6,5,4,2,1), \quad(6,5,5,1,1), \\
& (6,6,2,2,2), \quad(6,6,4,1,1), \quad(7,4,3,3,1), \quad(7,5,3,2,1), \quad(7,6,3,1,1), \\
& (7,7,2,1,1), \quad(8,3,3,2,2), \quad(8,4,3,2,1), \quad(8,5,2,2,1), \quad(9,5,2,1,1), \\
& (9,6,1,1,1),(10,3,2,2,1),(10,3,3,1,1),(10,4,2,1,1),(10,5,1,1,1), \\
& (11,3,2,1,1),(12,2,2,1,1),(12,3,1,1,1),(13,2,1,1,1),(14,1,1,1,1) .
\end{aligned}
$$


Conversely, we have

Theorem 2.2. For every multi-index $\boldsymbol{d}$ given in Theorem 2.1, there exists a specific triangulation $\mathcal{T}$ with index $\boldsymbol{d}$. Concrete shapes of $\mathcal{T}$ are as follows:

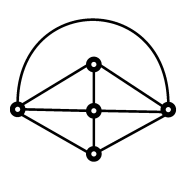

$(4,4,4,3,3)$

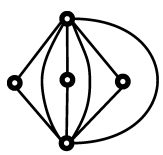

$(6,6,2,2,2)$

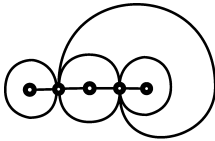

$(7,7,2,1,1)$

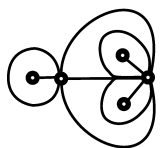

$(9,6,1,1,1)$

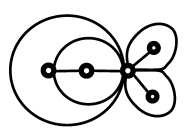

$(11,3,2,1,1)$

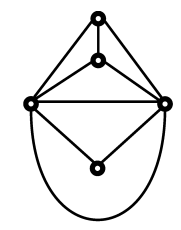

$(5,5,3,3,2)$

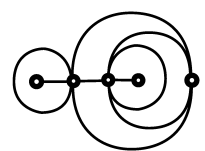

$(6,6,4,1,1)$

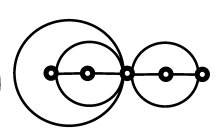

$(8,3,3,2,2)$

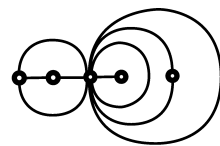

$(10,3,2,2,1)$

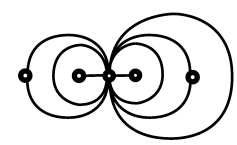

$(12,2,2,1,1)$

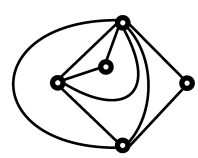

$(6,4,4,2,2)$

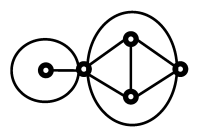

$(7,4,3,3,1)$

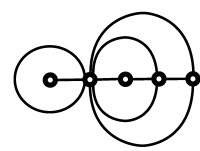

$(8,4,3,2,1)$

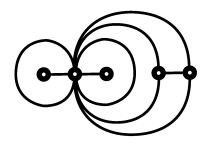

$(10,3,3,1,1)$

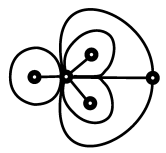

$(12,3,1,1,1)$

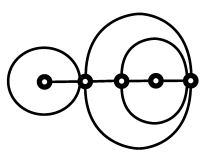

$(6,5,4,2,1)$

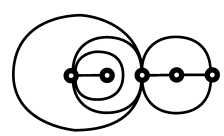

$(7,5,3,2,1)$

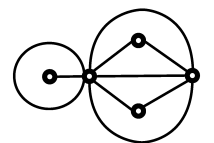

$(8,5,2,2,1)$

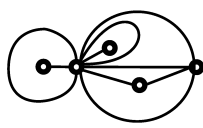

$(10,4,2,1,1)$

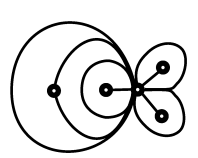

$(13,2,1,1,1)$

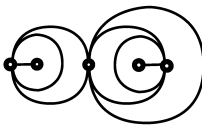

$(6,5,5,1,1)$

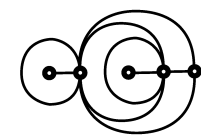

$(7,6,3,1,1)$

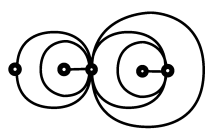

$(9,5,2,1,1)$

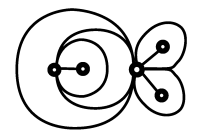

$(10,5,1,1,1)$

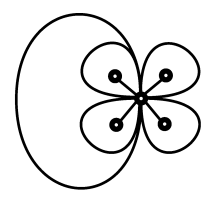

$(14,1,1,1,1)$

Fig 2.1

Here the symbol $\bigcirc$ designates a vertex and each (curvilinear) segment an edge. Thus all admissible indices of genus two are given in the table of Theorem 2.1.

Proof of Theorems 2.1 and 2.2 is given in Section 5. We do not discuss the uniqueness of the specific triangulation with a given index. In fact, the uniqueness breaks down in the case of $g=3$, while we empirically believe that it holds for $g \leq 2$ (up to orientation). 
§2.5. Classification of Stokes graphs of genus two

For a given abstract specific triangulation $\mathcal{T}$, we can make an abstract Stokes graph $\mathcal{S}$ so that the associated graph $\mathcal{G}^{*}$ coincides with $\mathcal{T}$ and vice versa. Combining this correspondence with Theorems 2.1 and 2.2, we have

Theorem 2.3. $\quad$ Let $\mathcal{S}$ be a Stokes graph of genus two and let $\boldsymbol{d}$ be the index of $\mathcal{S}$. Then $\boldsymbol{d}$ coincides with one of multi-indices given in Theorem 2.1. Conversely, for each multi-index d given in Theorem 2.1, there is an abstract Stokes graph $\mathcal{S}=(A, B, L)$ of index $\boldsymbol{d}$. Concrete shapes of $\mathcal{S}$ are given as follows:

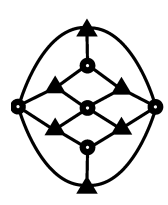

$(4,4,4,3,3)$

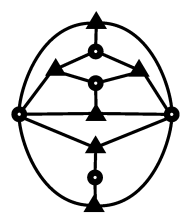

$(5,5,3,3,2)$

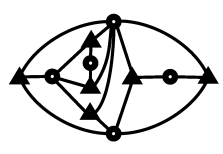

$(6,4,4,2,2)$

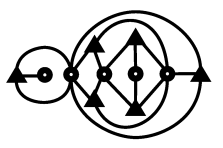

$(6,5,4,2,1)$

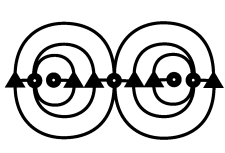

$(6,5,5,1,1)$

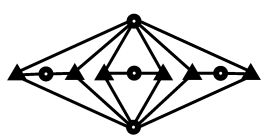

$(6,6,2,2,2)$

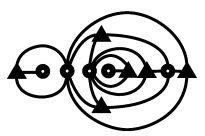

$(6,6,4,1,1)$

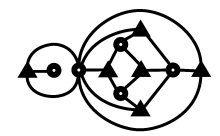

$(7,4,3,3,1)$

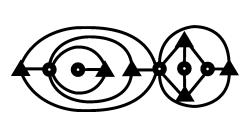

$(7,5,3,2,1)$

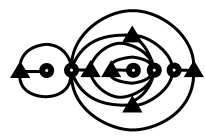

$(7,6,3,1,1)$

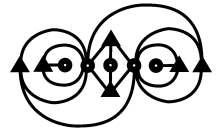

$(7,7,2,1,1)$

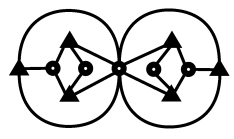

$(8,3,3,2,2)$

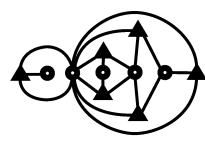

$(8,4,3,2,1)$

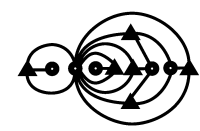

$(10,3,3,1,1)$

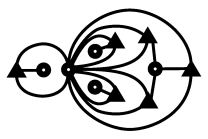

$(12,3,1,1,1)$

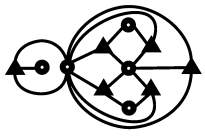

$(8,5,2,2,1)$

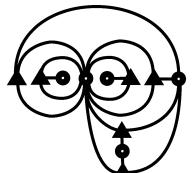

$(10,4,2,1,1)$

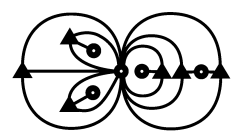

$(13,2,1,1,1)$

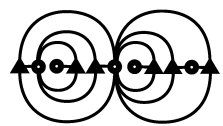

$(9,5,2,1,1)$

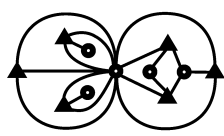

$(11,3,2,1,1)$

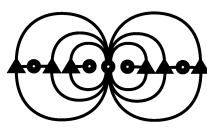

$(12,2,2,1,1)$

Fig 2.2

Here the symbol $\triangle$ designates a turning point (an element of $A$ ) and the symbol $\bigcirc$ designates a regular singular point (an element of $B$ ). 


\section{§3. Reduction of Triangulations of Genus $g$}

\section{§3.1. Shape of triangles of triangulations}

For a given specific triangulation $\mathcal{T}$ of the sphere, we take one triangle $f$ of $\mathcal{T}$. Let $V_{0}$ be the set of all points $f\left(t_{i}\right)(i=1,2,3)$ and let $n_{0}$ be the number of elements of $V_{0}$. Here $t_{i}$ are vertices of $\Delta$ (cf. Section 2.1). Topologically, there are four cases for the shape of the triangle $f(\bar{\Delta})$ :

- If $n_{0}=3$, we see that the sides $f\left(s_{j}\right)$ do not intersect each other by Condition 3 of specific triangulations and continuity of $f$. Thus the triangle $f(\bar{\Delta})$ has the shape topologically equivalent to the triangle shown in Fig. 3.1: I, which we call a triangle of type I.

- If $n_{0}=2$ and the sides $f\left(s_{j}\right)$ do not intersect each other, $f(\bar{\Delta})$ has the shape topologically equivalent to the triangle shown in Fig. 3.1: II, which we call a triangle of type II.

- If $n_{0}=2$ and two sides $f\left(s_{i}\right)$ and $f\left(s_{j}\right)$ have non-empty intersection, then we have $f\left(s_{i}\right)=f\left(s_{j}\right)$ by Condition 4 and thus the triangle $f(\bar{\Delta})$ has the shape topologically equivalent to the triangle shown in Fig. 3.1: III, which we call a triangle of type III.

- If $n_{0}=1$, then the triangle $f(\bar{\Delta})$ has the shape topologically equivalent to the triangle shown in Fig. 3.1: IV, which we call a triangle of type IV.

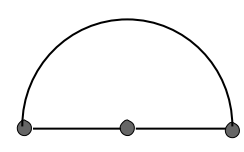

I

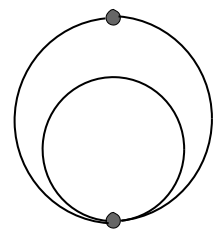

II

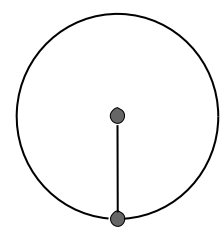

III

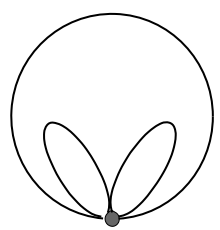

IV

Fig. 3.1: Four types of triangles.

Hence we may consider that $\mathcal{T}$ consists of triangles of these four types.

\section{$\S 3.2 . \quad$ Local configurations and reduction}

Let $\mathcal{T}=(V, E, F)$ be a specific triangulation of genus $g \geq 1$ of the sphere. Let $\boldsymbol{d}=\left(d_{1}, d_{2}, \ldots, d_{g+3}\right)$ denote the index of $\mathcal{T}$.

If $d_{g+3}=1$, there is a vertex $v_{1}$ of $\mathcal{T}$ with degree 1 . Hence there is a triangle $T_{0}$ of type III that has $v_{1}$ as one of its two vertices. The adjacent 
triangle $T_{1}$ via the loop-shaped side of $T_{0}$ should have type II, III or IV. If it has type III, then we see that $T_{0}$ and $T_{1}$ should cover the sphere and hence $g=0$. This contradicts our assumption. Thus $T_{1}$ is of type II or of type IV. If $T_{1}$ is of type II, then the (topological) boundary of $T_{0} \cup T_{1}$ consists of two sides of $T_{1}$. In this case, there are five possible configurations around $T_{0} \cup T_{1}$ (up to symmetry) which are shown in Figs. 3.2: (i)-(v). Here hatched regions consist of some triangles. If $T_{1}$ is of type IV, there are two possible local configurations shown in Figs. 3.2: (vi), (vii).

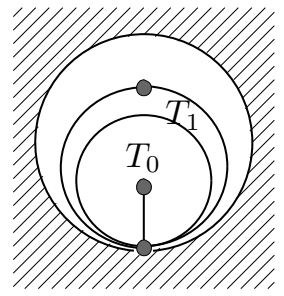

(i)

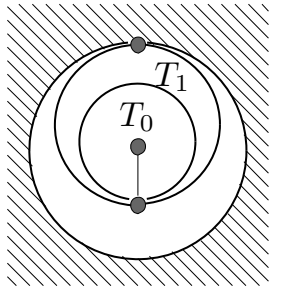

(ii)

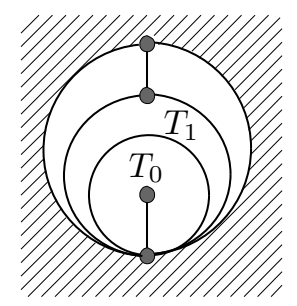

(iii)

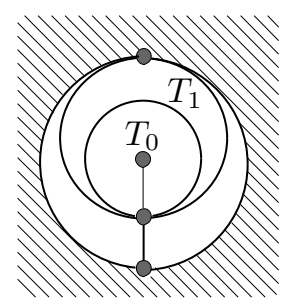

(iv)

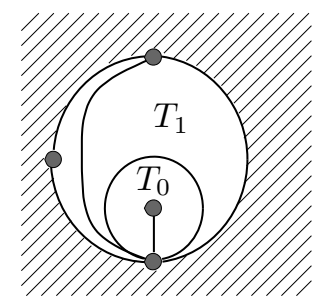

(

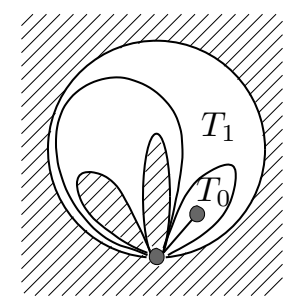

(vi)

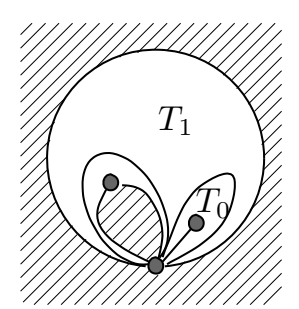

(vii)

Fig. 3.2: Local configurations near a vertex of degree 1.

Let us remove $T_{0}, T_{1}$ and $v_{1}$ from these configurations. There appear "holes" (double hatched regions in Figs. 3.3) surrounded by two edges that had formed sides of $T_{1}$. We remove these holes by contracting the edges to one new edge. ("Close" the holes by zipping up the two edges.) Then we have new triangle(s) shown in the right-most figures in Fig. 3.3. Note that these procedures do not affect triangles located in hatched regions. Comparing the final configurations with the original ones, we see that the numbers of triangles, edges and vertices decrease 2, 3 and 1 , respectively. This intuitive illustration can be stated by using the terminologies of the graph theory. For example, we consider the case of Fig. 3.2: (i). Let $e_{1}$ be the edge incident with $v_{1}$ and let $e_{2}$ be another edge(side) of $T_{0}$. Let $v_{2}$ denote another vertex of $T_{0}$ and let $T_{2}$ denote the triangle adjacent to $T_{0} \cup T_{1}$ via edges(sides) $e_{3}, e_{4}$ of $T_{1}$. We set 

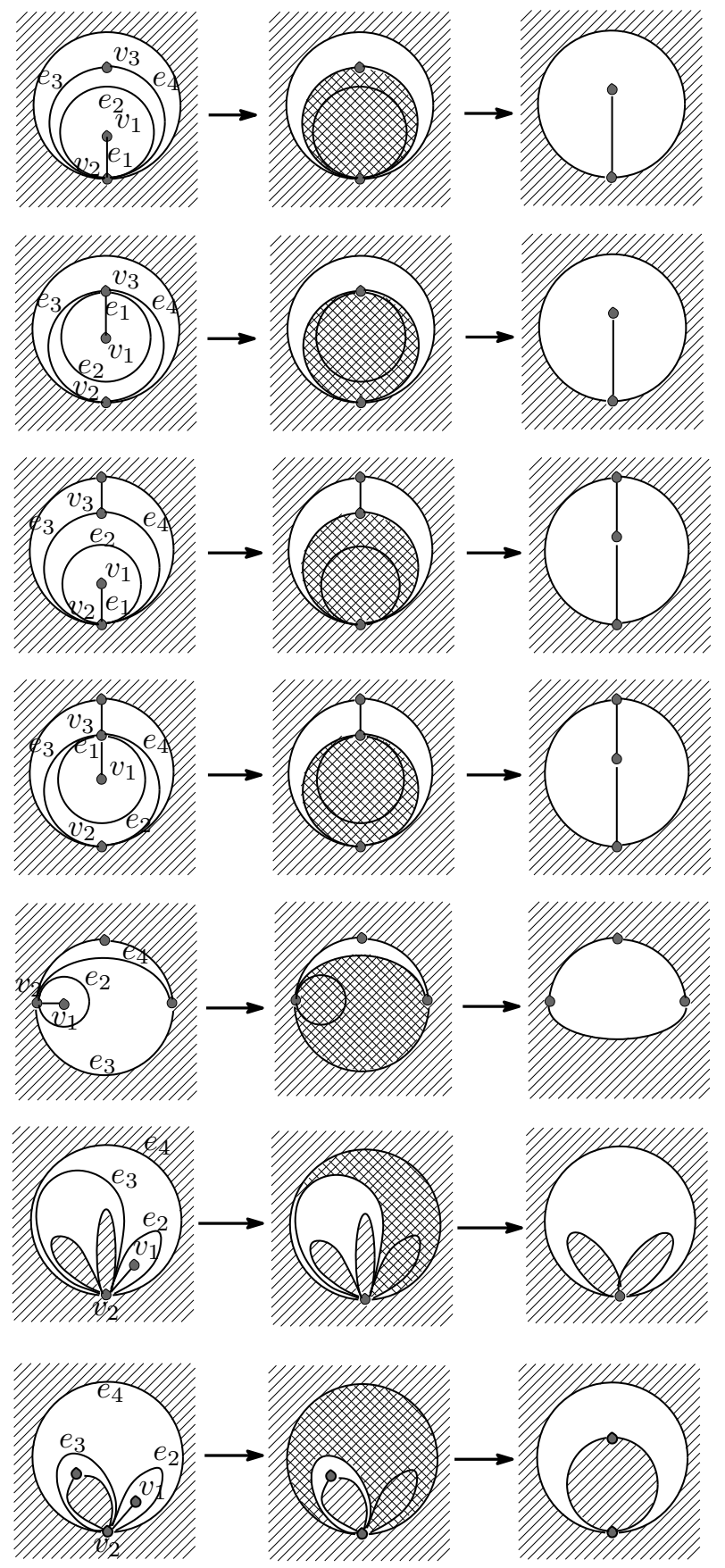

Fig. 3.3: Reduction of local configurations. 
$V^{\prime}=V-\left\{v_{1}\right\}, E^{\prime}=E-\left\{e_{1}, e_{2}, e_{3}\right\}$ and $F^{\prime}=\left(F-\left\{T_{0}, T_{1}, T_{2}\right\}\right) \cup\left\{T_{2}^{\prime}\right\}$ with $T_{2}^{\prime}=T_{2} \cup T_{1} \cup T_{0} \cup\left\{v_{3}\right\}$. Thus we have obtained a specific triangulation $\mathcal{T}^{\prime}$ of genus $g-1$ from $\mathcal{T}$. Other cases can be discussed in similar manners. Next we consider the case where $d_{g+3}=2$. There exists a vertex $v_{1}$ of $\mathcal{T}$ with degree two. Hence there are two edges $e_{1}$ and $e_{2}$ which are incident with $v_{1}$. Let $v_{2}$ (resp. $v_{3}$ ) be another vertex incident with $e_{1}$ (resp. $e_{2}$ ). If $v_{2}=v_{3}$, there are two loop-shaped edges(sides) $e_{3}$ and $e_{4}$ which have $v_{2}$ as the starting point and the endpoint. Thus $v_{1}$ is a common vertex of two triangles $T_{1}$ and $T_{2}$ of type II. Here $T_{1}$ (resp. $T_{2}$ ) is a triangle incident with $e_{1}, e_{2}, e_{3}\left(\right.$ resp. $e_{1}, e_{2}, e_{4}$ ) (see Fig. 3.4: (i)).

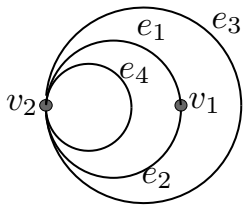

(i)

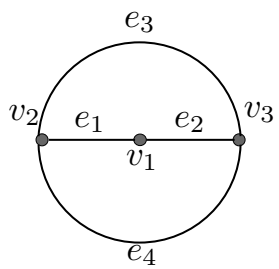

(ii)

Fig. 3.4

If $v_{2} \neq v_{3}$, then there are two edges $e_{3}$ and $e_{4}$ which are incident with $v_{2}$ and $v_{3}$. Thus $v_{1}$ is a common vertex of two triangles $T_{1}$ and $T_{2}$ of type I. Here $T_{1}$ and $T_{2}$ are defined similarly as shown in Fig. 3.4: (ii). In both cases, $T_{1} \cup T_{2}$ is surrounded by $e_{3}$ and $e_{4}$. Hence there are four possible local configurations around $v_{1}$ shown in Figs. 3.5: (i)-(iv):

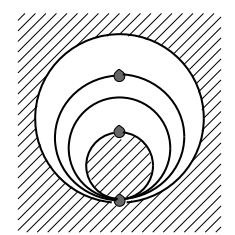

(i)

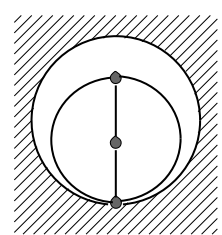

(ii)

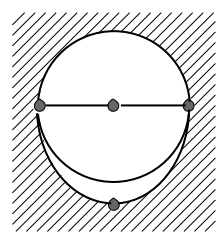

(iii)

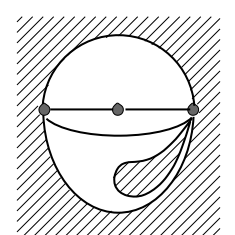

(iv)

Fig. 3.5: Local configurations near a vertex of degree 2 .

Now we remove $T_{1}, T_{2}, e_{1}, e_{2}$ and $v_{1}$ from $\mathcal{T}$ and contract two edges $e_{3}$ and $e_{4}$ to one new edge $e_{3}^{\prime}$. For every configuration given in Fig. 3.5, this procedure does not affect the hatched region(s) and we have a specific triangulation $\mathcal{T}^{\prime}$ of genus $g-1$. See Fig. 3.6. 

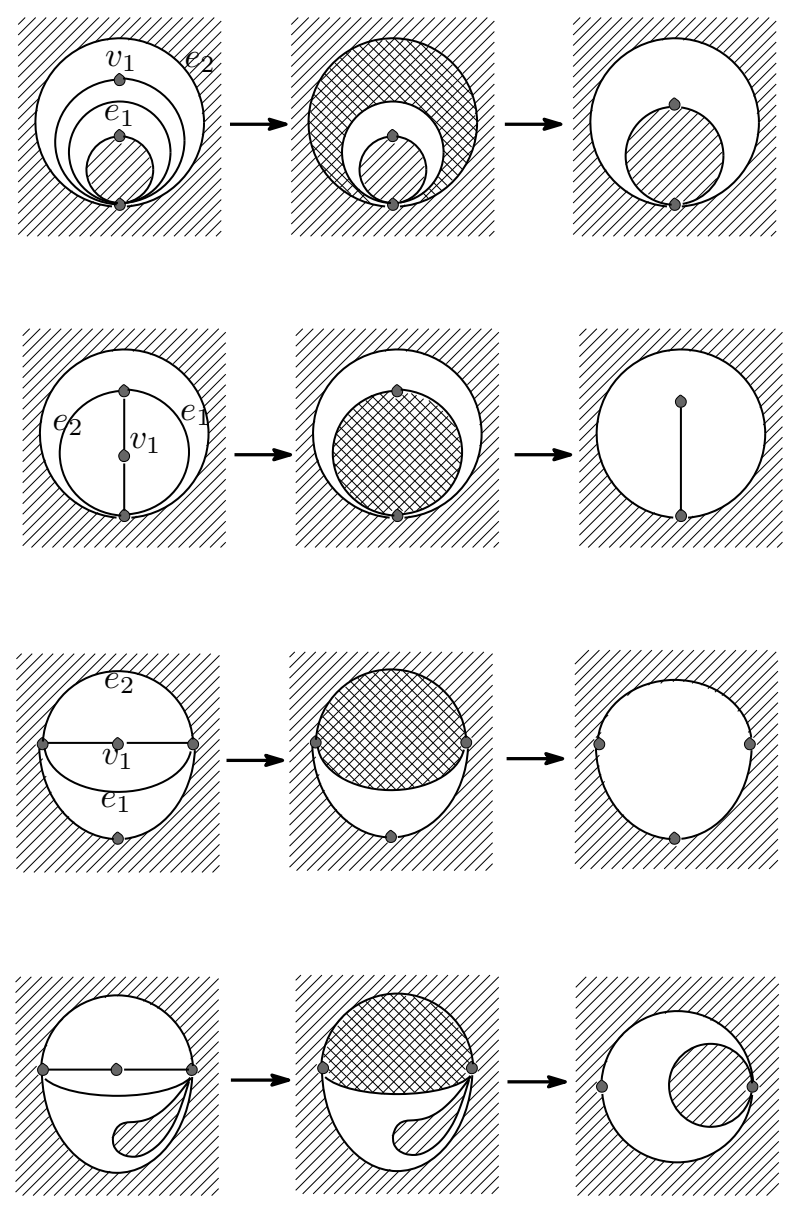

Fig. 3.6: Reduction of local configurations with a vertex of degree 2 .

Finally we consider the case where $d_{g+3}=3$. There is a vertex $v_{0}$ of $\mathcal{T}$ of degree three. Let $e_{1}, e_{2}, e_{3}$ be edges incident with $v_{0}$. Let $v_{j}$ denote the vertex of $e_{j}$ different from $v_{0}(j=1,2,3)$. There are three cases. If $v_{1}, v_{2}, v_{3}$ are mutually distinct, $v_{0}$ is a common vertex of three triangles $T_{1}, T_{2}, T_{3}$ of type I. Here we set $T_{1}=\triangle v_{0} v_{2} v_{3}, T_{2}=\triangle v_{0} v_{3} v_{1}$ and $T_{3}=\triangle v_{0} v_{1} v_{2}$ (cf. Fig. 3.7: (i)). If $v_{1}=v_{2} \neq v_{3}, v_{0}$ is a common vertex of three triangles and one of them is not incident with $v_{3}$. We denote it by $T_{3}$. Other two triangles are denoted by $T_{1}$ and $T_{2}$ (cf. Fig. 3.7: (ii)). Note that $T_{3}$ is surrounded by edges $v_{0} v_{1}, v_{1} v_{1}$ and $v_{1} v_{0}$ and $v_{1} v_{1}$ is a loop. Hence $T_{3}$ is of type II, while $T_{1}$ and $T_{2}$ are of type I. If $v_{1}=v_{2}=v_{3}, v_{0}$ is a common vertex of two triangles $T_{1}, T_{2}$ of type II and of a triangle $T_{3}$ of type I (cf. Fig. 3.7: (iii)). 


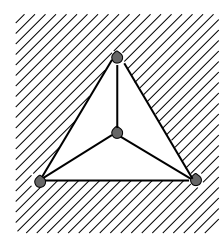

(i)

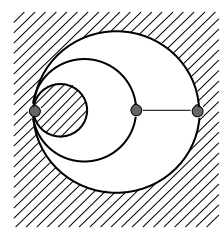

(ii)

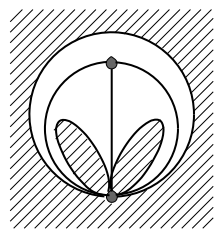

(iii)

Fig. 3.7: Local configurations near a vertex of degree 3 .

In each case, we can remove $T_{1}, T_{2}, v_{0}$ and contract relating edges to obtain a specific triangulation of genus $g-1$. This procedure is illustrated in Fig. 3.8.
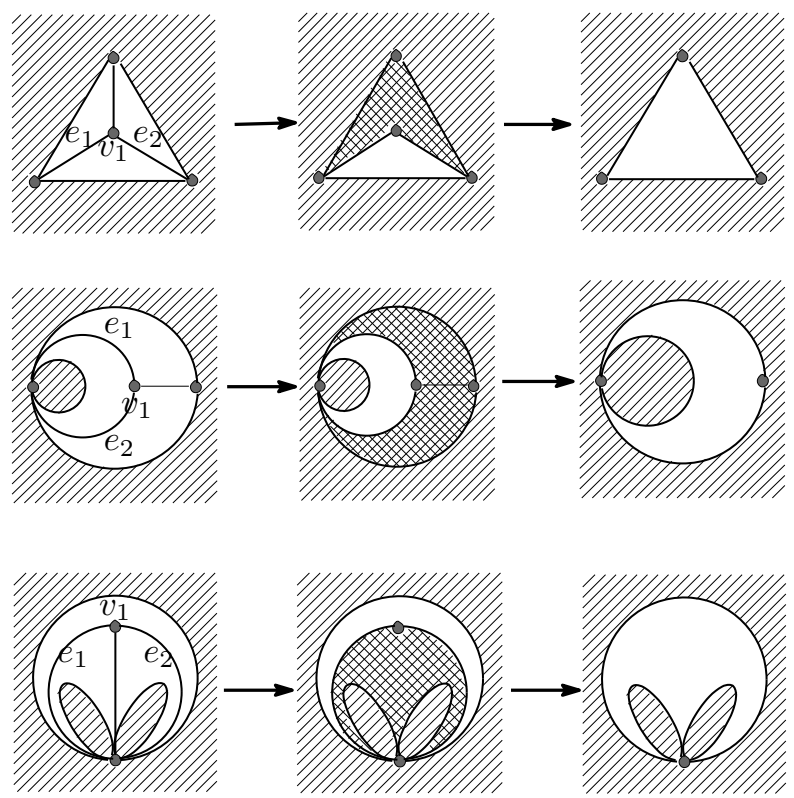

Fig. 3.8: Reduction of local configurations with a vertex of degree 3.

The procedure of obtaining $\mathcal{T}^{\prime}$ from $\mathcal{T}$ (or $\mathcal{T}^{\prime}$ itself) is called a reduction (of $\mathcal{T})$. Thus we have obtained the following

Theorem 3.1. $\quad$ Let $\mathcal{T}$ be a specific triangulation of genus $g \geq 1$ of the sphere and let $\boldsymbol{d}=\left(d_{1}, d_{2}, \ldots, d_{g+3}\right)$ be the index of $\mathcal{T}$. If $d_{g+3} \leq 3$, then there is at least one specific triangulation $\mathcal{T}^{\prime}$ of genus $g-1$ which is obtained by the reduction of $\mathcal{T}$. 


\section{§4. Augmentation of Triangles}

\section{§4.1. Two-triangle cells}

We consider procedure reciprocal to the reduction discussed in the preceding section. The reduction consists of removing a pair of triangles, a vertex and contracting related edges. Thus the reciprocal procedure should consist of blow up one or two edge(s) (see Section 4.2) and squeeze a pair of triangles there so that the number of triangles, edges and vertices increase 2, 3 and 1 , respectively. Blow up of one (resp. two) edge(s) yields a dilateral (resp. quadrangle). There are three types of making a dilateral or a quadrangle by taking union of two triangles. First one is obtained by glueing two sides of two triangles of type I each other(cf. Fig. 4.1: A). Second one is made by covering the "hole" of a triangle of type II by a triangle of type III (cf. Fig 4.1: B). Third one is obtained by glueing one side of a triangle of type I with one side of another triangle of type I (cf. Fig. 4.1: C). These pairs are called two triangle cell of type $\mathrm{A}, \mathrm{B}$, and $\mathrm{C}$, respectively.

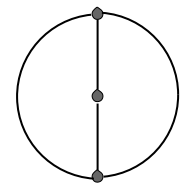

type A

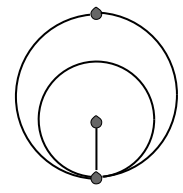

type B

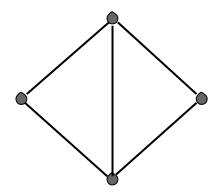

type $\mathrm{C}$

Fig. 4.1: Two-triangle cells.

\section{$\S 4.2$. Blow up}

Let $\mathcal{T}$ be a specific triangulation of genus $g$ of the sphere. Let $T_{0}$ be a triangle (face) of $\mathcal{T}$.

(i) We consider the case where $T_{0}$ is a triangle of type I. Let $e_{1}, e_{2}, e_{3}$ denote the sides of $T_{0}$. First we take an edge, say $e_{1}$, and make a copy of it. We dislocate the copy slightly to one direction transversal to $e_{1}$ by preserving its incident vertices. Then we have a new edge $e_{1}^{\prime}$. We consider a dilateral $D$ surrounded by $e_{1}$ and $e_{1}^{\prime}$ (see the double hatched region of Fig. 4.2: (i)).

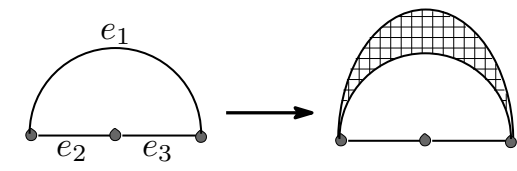

(i)

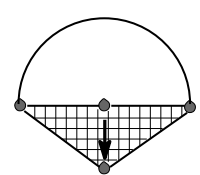

(ii)

Fig. 4.2 
Now we replace $D$ by a two triangle cell of type A or of type B (cf. Fig. 4.3). This procedure yields two specific triangulations $\hat{T}$ and $\hat{T}^{\prime}$ of genus $g+1$. We call this procedure one-edge blow up of $T_{0}$ (or of $\mathcal{T}$ ) with the center $e_{1}$. We also call $\hat{T}$ and $\hat{T}^{\prime}$ blow ups of $\mathcal{T}$. Next we take two edges, say $e_{2}$ and $e_{3}$. Let $v_{1}$ denote the vertex incident with $e_{2}$ and $e_{3}$. Other vertices which are incident with $e_{2}$ and with $e_{3}$ are denoted by $v_{2}$ and $v_{3}$, respectively. We make copies of $e_{2}, e_{3}$ and $v_{1}$ and dislocate them slightly preserving $v_{2}$ and $v_{3}$. Then we have new edges $e_{2}^{\prime}, e_{3}^{\prime}$ and a new vertex $v_{1}^{\prime}$. There is a quadrangle $Q$ surrounded by $e_{2}, e_{3}, e_{2}^{\prime}$ and $e_{3}^{\prime}$ (see the double hatched region of Fig. 4.2: (ii)). Now we replace $Q$ by a two triangle cell of type $\mathrm{C}$ and get a specific triangulation $\hat{T}^{\prime \prime}$ of genus $g+1$ (cf. Fig. 4.3). We call this procedure two-edge blow up of $T_{0}$ (or of $\mathcal{T}$ ) with the centers $e_{2}$ and $e_{3}$. The specific triangulation $\hat{T}^{\prime \prime}$ is also called a blow up of $\mathcal{T}$.

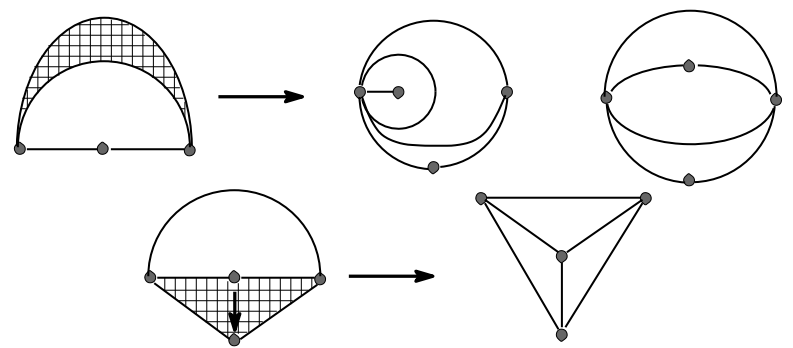

Fig. 4.3

In the cases where $T_{0}$ is of type II, III or IV, we can define one-edge blow up and two-edge blow up in similar ways. We only give figures to illustrate these procedures.

(ii) The case of type II:
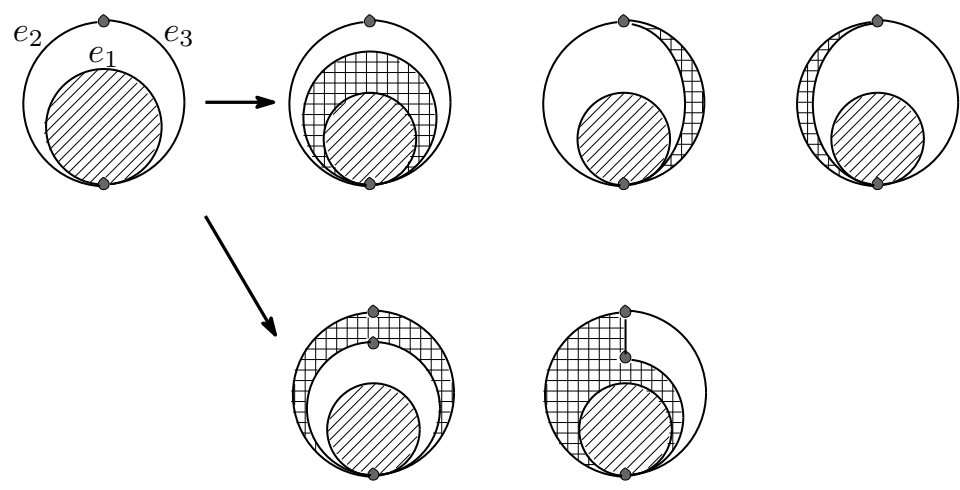

Fig. 4.4: Making dilaterals or quadrangles (double hatched regions). 


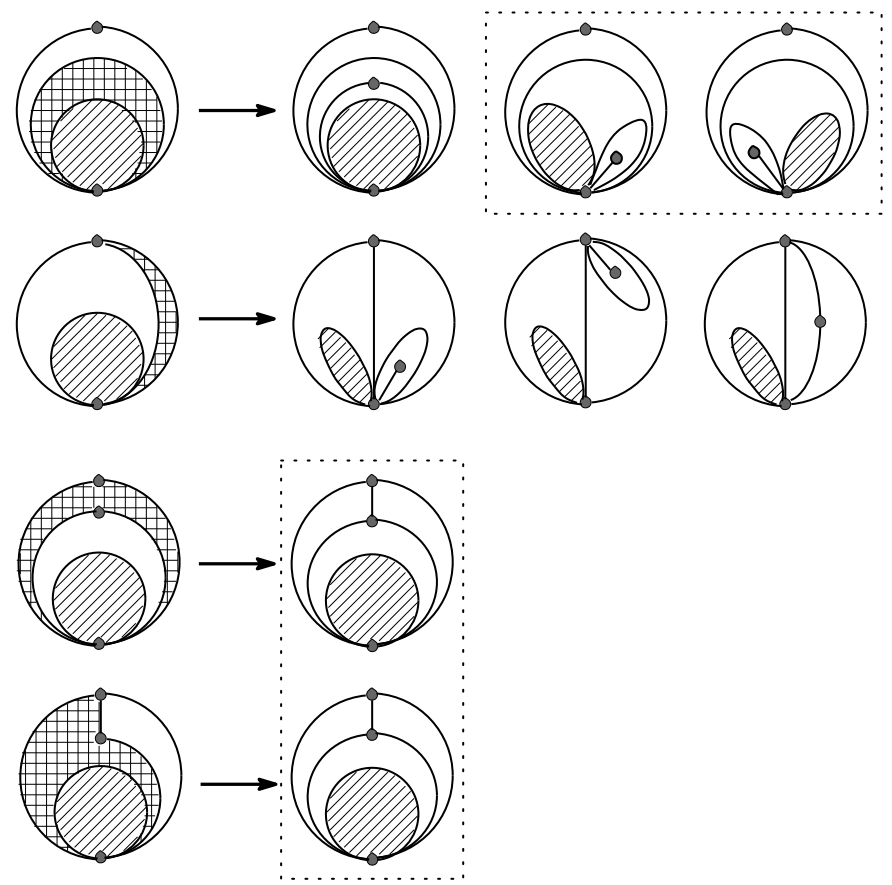

Fig. 4.5: Blow up yields 6 configurations. Configurations enclosed by dotted-lined squares are the same (up to symmetry).

(iii) The case of type III:

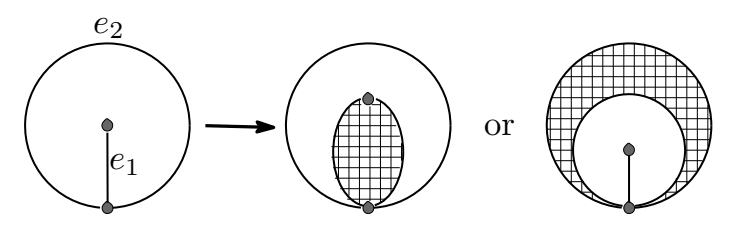

Fig. 4.6: Making dilaterals (double hatched regions). 


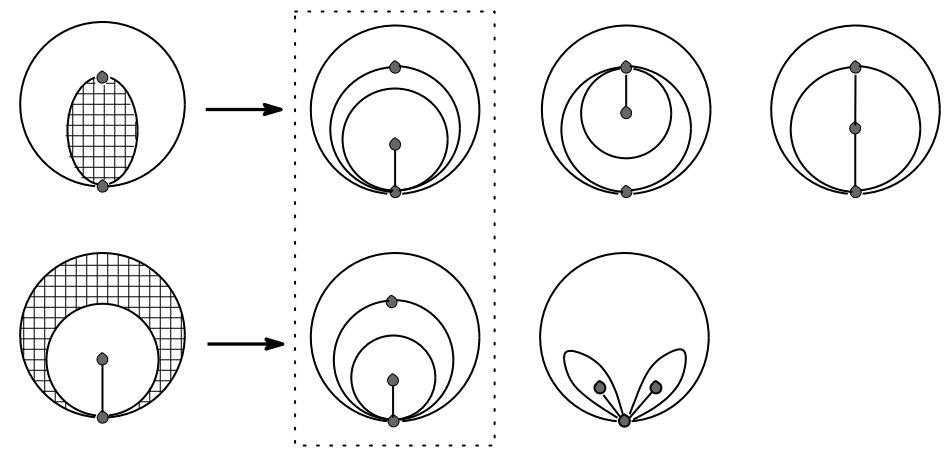

Fig. 4.7: Blow up yields 4 configurations.

Note that the two-edge blow up with the centers $e_{1}, e_{2}$ yields one of the configuration given in Fig. 4.3. Thus we do not need the two-edge blow up for triangles of type III.

(iv) The case of type IV:
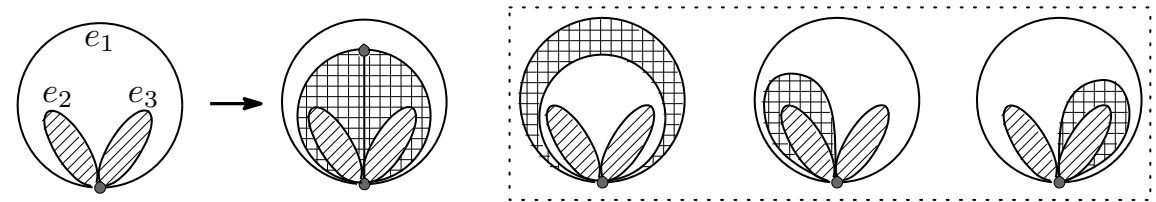

Fig. 4.8: Making a quadrangle or dilaterals (double hatched regions).
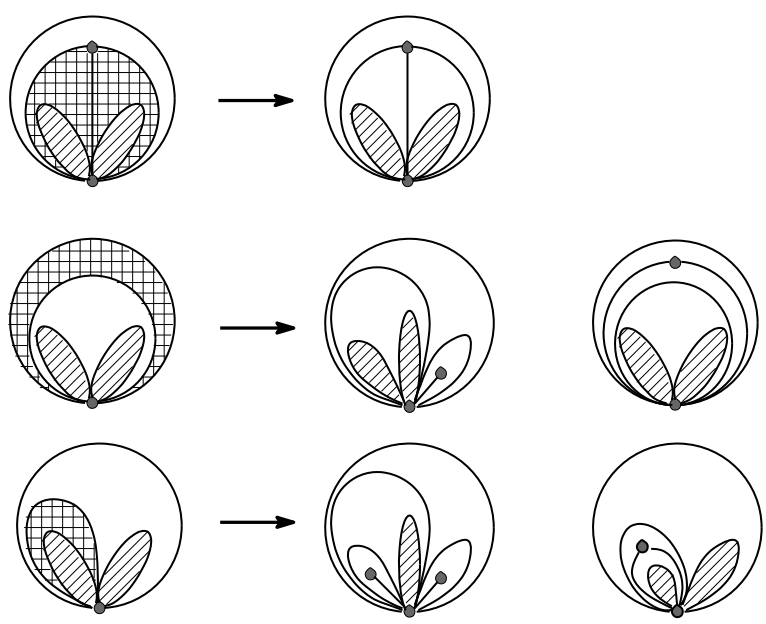

Fig. 4.9: Blow up yields 5 configurations. 
Hence we have

Theorem 4.1. $\quad$ Let $\mathcal{T}$ be a specific triangulation of genus $g \geq 0$ of the sphere. Then there are specific triangulations of genus $g+1$ which are obtained as a one-edge blow up or a two-edge blow up of $\mathcal{T}$.

By the definition of the reduction, we have

Theorem 4.2. $\quad$ Let $\mathcal{T}$ and $\mathcal{T}^{\prime}$ be specific triangulations of genus $g(\geq 1)$ and of $g-1$ of the sphere, respectively. Let $\boldsymbol{d}=\left(d_{1}, d_{2}, \ldots, d_{g+3}\right)$ denote the index of $\mathcal{T}$. Suppose that $d_{g+3} \leq 3$ and $\mathcal{T}^{\prime}$ is a reduction of $\mathcal{T}$. Then $\mathcal{T}$ is a blow up of $\mathcal{T}^{\prime}$.

\section{§5. Proof of Theorems 2.1 and 2.2}

Let $\mathcal{T}$ be a specific triangulation of genus two of the sphere and let $\boldsymbol{d}=$ $\left(d_{1}, d_{2}, d_{3}, d_{4}, d_{5}\right)$ denote the index of $\mathcal{T}$. Since $\sum_{k=0}^{5} d_{k}=18$ and $d_{1} \geq d_{2} \geq$ $\cdots \geq d_{5}$, we have $d_{5} \leq 3$. It follows from Theorems 3.1 and 4.2 that $\mathcal{T}$ is a blow up of some specific triangulation of genus one. We know that there are six types of specific triangulations of genus one [5], [4]. The indices of these six triangulations are

$$
\begin{array}{llll}
(3,3,3,3), & (4,4,2,2), & (9,1,1,1), \\
(8,2,1,1), & (5,5,1,1), & (6,3,2,1)
\end{array}
$$

and configurations of triangles are given as follows:

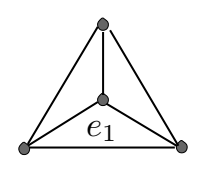

$(3,3,3,3)$

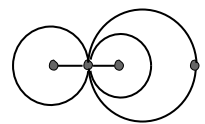

$(8,2,1,1)$

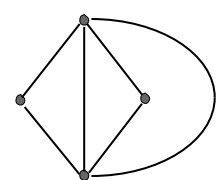

$(4,4,2,2)$

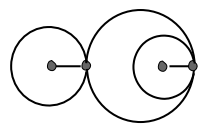

$(5,5,1,1)$

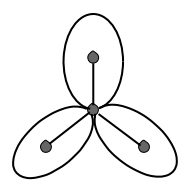

$(9,1,1,1)$

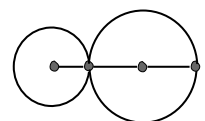

$(6,3,2,1)$

Fig. 5.1: Six types of specific triangulations of genus one.

Thus $\mathcal{T}$ is a blow up of a specific triangulation with one of these indices. We consider all possible blow ups of triangulations of genus one. 


\section{$\S 5.1$. The case of $(3,3,3,3)$}

Let us consider a specific triangulation $\mathcal{T}^{\prime}$ of genus 1 with index $(3,3,3,3)$. We choose one edge $e_{1}$ of $\mathcal{T}^{\prime}$ and take one-edge blow ups with the center $e_{1}$ (see Fig. 5.2 below).

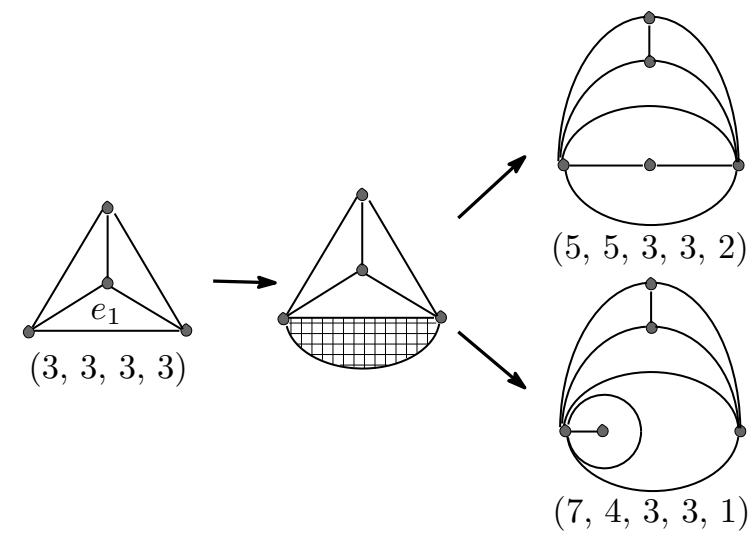

Fig. 5.2: One-edge blow up with the center $e_{1}$.

Then we have two different types of specific triangulations of genus two with indices $(5,5,3,3,2)$ and $(7,4,3,3,1)$, respectively. Since all edges of $\mathcal{T}^{\prime}$ are symmetric with respect to this procedure, we have these two types of specific triangulations of genus two by the one-edge blow-up in the case of $(3,3,3,3)$.

Next we choose one vertex $v_{1}$ of $\mathcal{T}^{\prime}$ and two edges $e_{1}, e_{2}$ which are incident with $v_{1}$. Then the two-edge blow up with the centers $e_{1}, e_{2}$ yields a specific triangulation of genus two with index $(4,4,4,3,3)$ as is shown in the following figure:

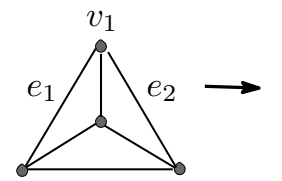

$(3,3,3,3)$

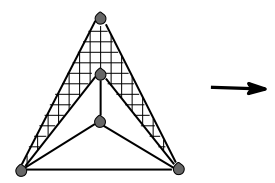

Fig. 5.3: Two-edge blow up with the centers $e_{1}, e_{2}$.

This procedure is also symmetric with respect to the choice of $v_{1}, e_{1}, e_{2}$.

Hence we have three admissible indices of genus two by the blow up of $(3,3,3,3)$ :

$$
(5,5,3,3,2),(7,4,3,3,1),(4,4,4,3,3)
$$




\section{$\S 5.2$. The case of $(4,4,2,2)$}

Let $\mathcal{T}^{\prime}$ be a specific triangulation of genus one with index $(4,4,2,2)$. Let $v_{1}, v_{2}$ be vertices of $\mathcal{T}^{\prime}$ of degree 4 and $v_{3}, v_{4}$ vertices of degree 2 . Let $e_{1}$ be an edge incident with $v_{1}, v_{2}$. Let $e_{2}$ and $e_{3}$ be edges incident with $v_{1}, v_{4}$ and $v_{2}$, $v_{4}$, respectively. There are two ways (up to symmetry) of the one-edge blow up. That is, the one edge blow up with the center $e_{1}$ and with center $e_{2}$. The former yields admissible indices $(6,6,2,2,2),(8,5,2,2,1)$ and the latter $(8,4,3,2,1),(6,4,4,2,2),(6,5,4,2,1)$ (see Figs. 5.4 and 5.5).

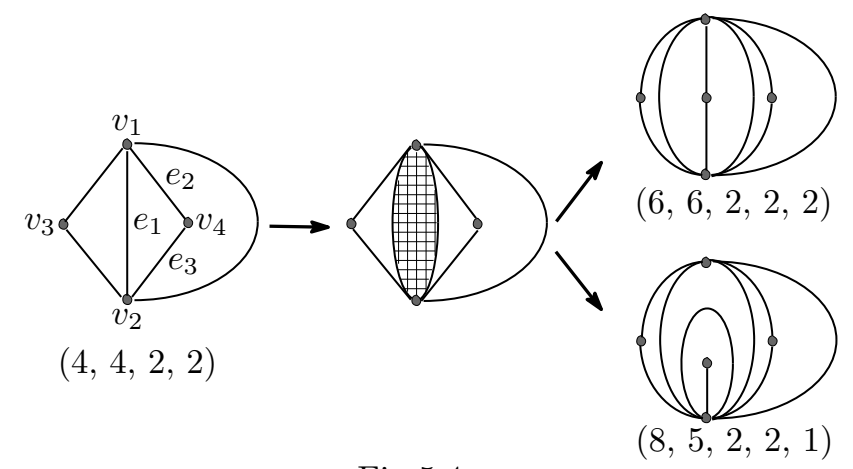

Fig 5.4

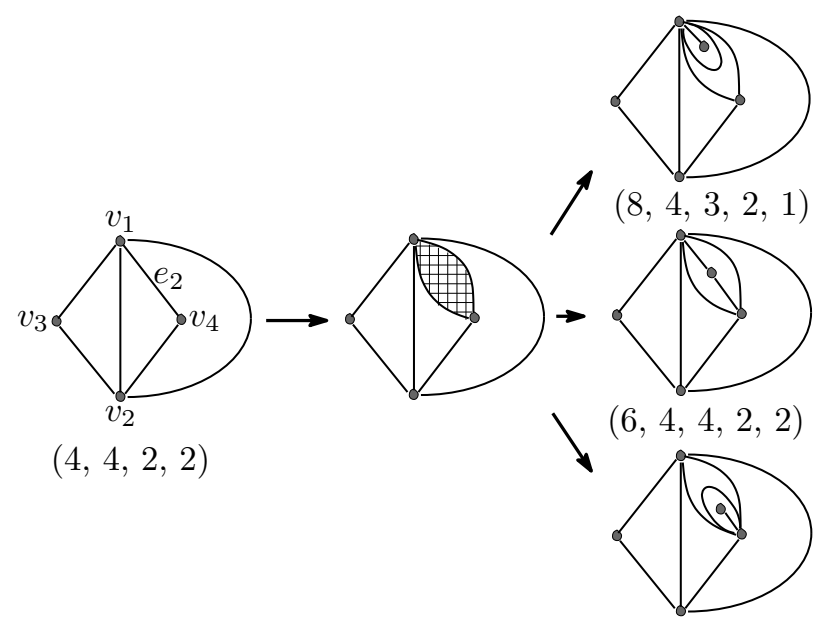

Fig. 5.5

$(6,5,4,2,1)$

On the other hand, there are two ways (up to symmetry) of the two-edge blow up. That is, the blow up with the center $e_{2}, e_{3}$ and with $e_{1}, e_{2}$. Both cases yield the same admissible index $(5,5,3,3,2)$ (see Figs. 5.6 and 5.7). This index has already been found in the preceding section. 


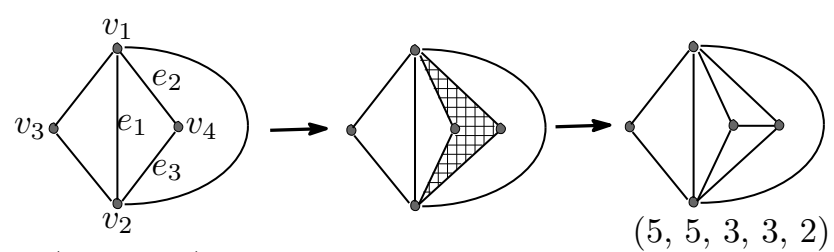

$(4,4,2,2)$

Fig. 5.6

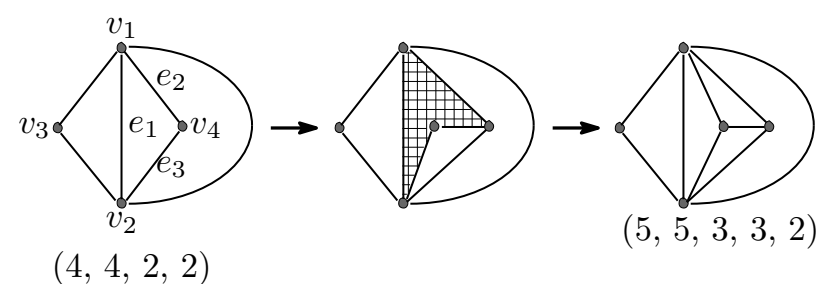

Fig. 5.7

Hence we have five admissible indices of genus two by the blow up of $(4,4,2,2)$ :

$(6,6,2,2,2),(8,5,2,2,1),(8,4,3,2,1)$,

$(6,4,4,2,2),(6,5,4,2,1)$.

\section{$\S 5.3$. The case of $(9,1,1,1)$}

Let $\mathcal{T}^{\prime}$ be a specific triangulation of genus one with index $(9,1,1,1)$. Let $v_{1}$ be the vertex of $\mathcal{T}^{\prime}$ of degree 9 and $v_{2}, v_{3}, v_{4}$ vertices of degree 1 . Let $e_{4}, e_{5}$ and $e_{6}$ denote edges of $\mathcal{T}^{\prime}$ incident with $v_{1}, v_{2}$, with $v_{1}, v_{3}$ and with $v_{1}$, $v_{4}$, respectively. There are three other edges, which are denoted by $e_{1}, e_{2}$ and $e_{3}$. Note that $e_{1}, e_{2}$ and $e_{3}$ are incident with only one vertex $v_{1}$. There are two possible way up to symmetry. One-edge blow up with the center $e_{1}$ yields two admissible indices $(14,1,1,1,1)$ and $(13,2,1,1,1)$ and that with the center $e_{4}$ yields three admissible indices $(10,5,1,1,1),(13,2,1,1,1)$, $(11,3,2,1,1)$ (see Figs. 5.8 and 5.9). 


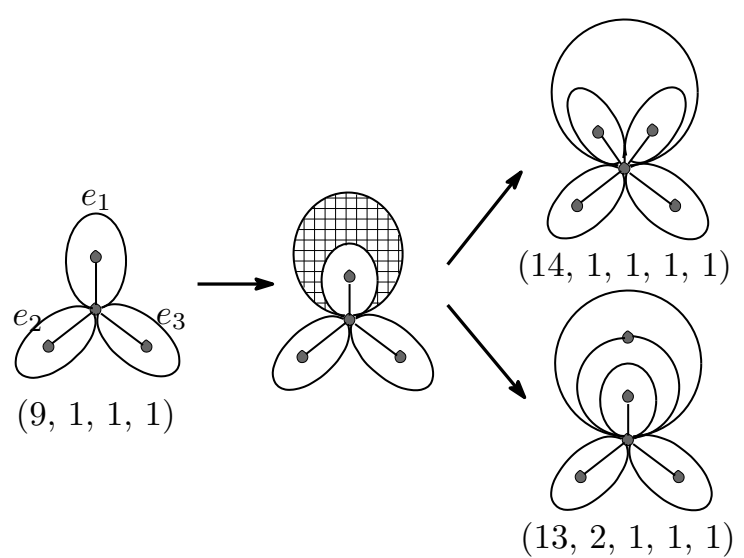

Fig. 5.8

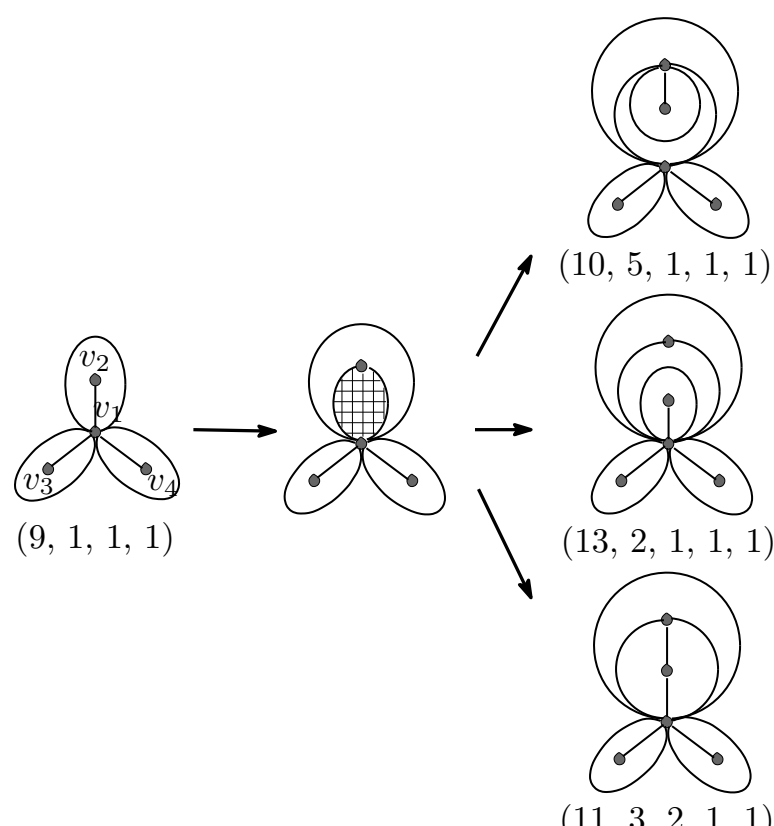

Fig. 5.9

The index $(13,2,1,1,1)$ has been already appeared in Fig. 5.8. The two-edge blow up of $\mathcal{T}^{\prime}$ is unique up to symmetry. The blow up with the centers $e_{2}, e_{3}$ yields an admissible index $(12,3,1,1,1)$ (see Fig. 5.10). 


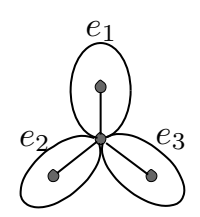

$(9,1,1,1)$

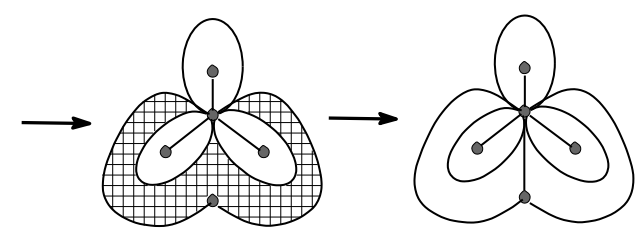

$(12,3,1,1,1)$

Fig. 5.10

Hence we have five admissible indices of genus two by the blow up of $(9,1,1,1)$ :

$$
\begin{aligned}
& (14,1,1,1,1),(13,2,1,1,1),(10,5,1,1,1), \\
& (11,3,2,1,1),(12,3,1,1,1) .
\end{aligned}
$$

\section{$\S 5.4$. The case of $(8,2,1,1)$}

Let $\mathcal{T}^{\prime}$ be a specific triangulation of genus one with index $(8,2,1,1)$. Let $v_{1}$ be the vertex of $\mathcal{T}^{\prime}$ of degree $8, v_{2}$ the vertex of degree 2 and $v_{3}, v_{4}$ vertices of degree 1 . Let $e_{1}, e_{2}$ denote edges incident with only one vertex $v_{1}$. Let $e_{3}$ and $e_{4}$ be edges incident with $v_{1}, v_{3}$ and with $v_{1}, v_{4}$, respectively. Two edges incident with $v_{1}, v_{2}$ are denoted by $e_{5}, e_{6}$. There are three possible ways up to symmetry for the one-edge blow up. By the one-edge blow up with the center $e_{1}$, we obtain two admissible indices $(12,2,2,1,1)$ and $(13,2,1,1,1)$ but the latter has appeared in Section 5.3.

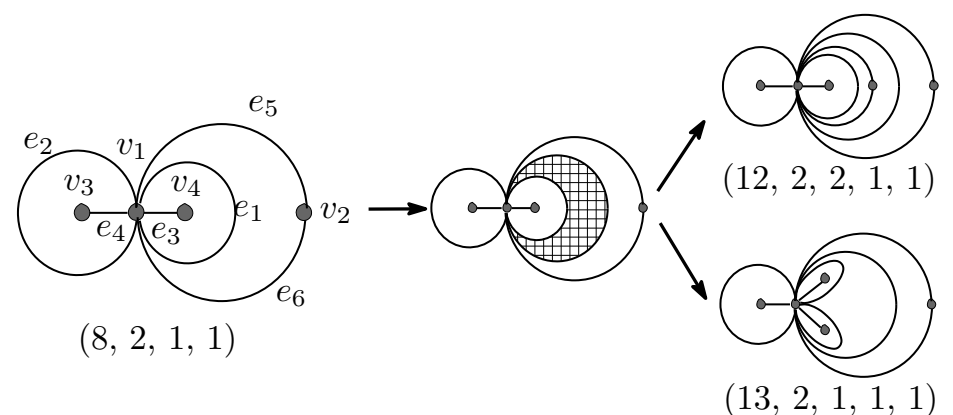

Fig. 5.11

The one-edge blow up with the center $e_{4}$ yields three admissible indices $(12,2$, $2,1,1),(9,5,2,1,1),(10,3,2,2,1)$. The first one has already been obtained in Fig. 5.11. 


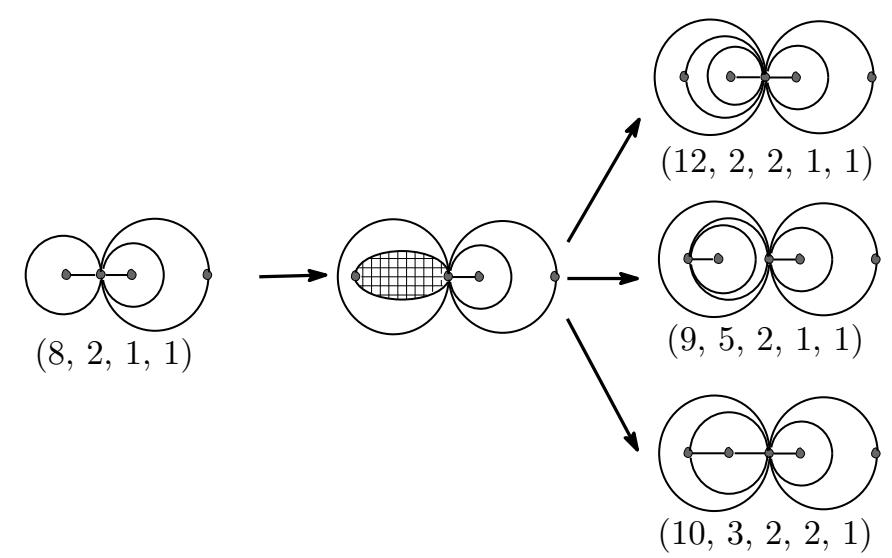

Fig. 5.12

By the one-edge blow up with the center $e_{5}$, we have three admissible indices $(12,3,1,1,1),(9,6,1,1,1),(10,4,2,1,1)$. The first one has been found in Section 5.3.

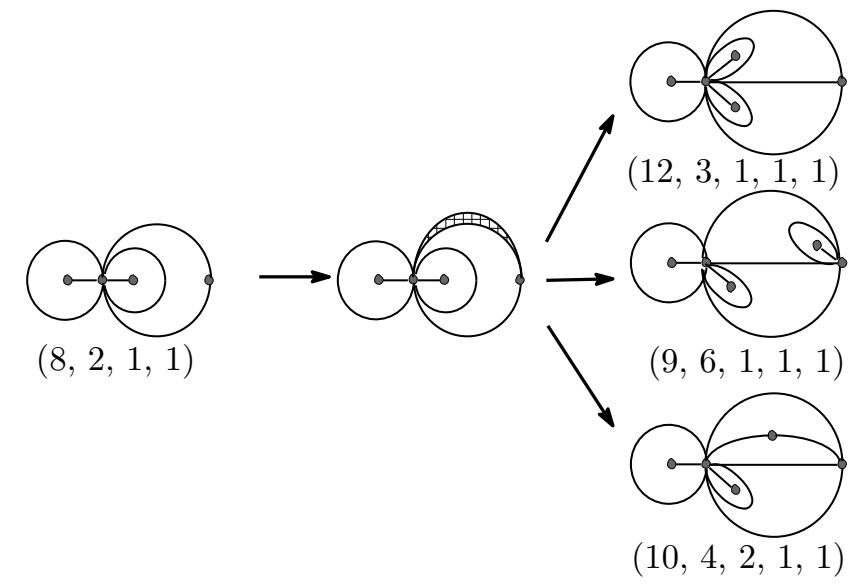

Fig. 5.13

The two-edge blow up is unique up to symmetry. Taking the blow up with the centers $e_{5}, e_{6}$ yields an admissible index $(10,3,3,1,1)$.

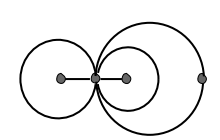

$(8,2,1,1)$

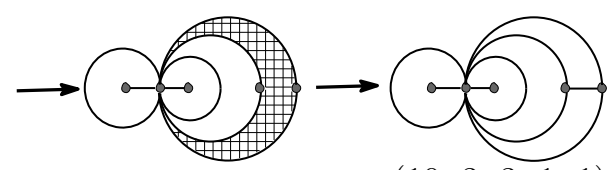

$(10,3,3,1,1)$

Fig. 5.14 
Hence we have six admissible indices:

$(12,2,2,1,1),(9,5,2,1,1),(10,3,2,2,1)$,

$(9,6,1,1,1),(10,4,2,1,1),(10,3,3,1,1)$.

\section{$\S 5.5$. The case of $(5,5,1,1)$}

Let $\mathcal{T}^{\prime}$ be a specific triangulation of genus one with index $(5,5,1,1)$. Let $v_{1}, v_{2}$ be the vertex of $\mathcal{T}^{\prime}$ of degree 5 . There are two edges incident with $v_{1}, v_{2}$. We denote them by $e_{1}, e_{2}$. There are two other edges incident with $v_{1}$ (resp. $v_{2}$ ): One of them is a loop (incident only with vertex $v_{1}\left(\right.$ resp. $\left.v_{2}\right)$ ), which is denoted by $e_{3}$ (resp. $\left.e_{4}\right)$ and the other is denoted by $e_{5}$ (resp. $e_{6}$ ). Edge $e_{5}\left(\right.$ resp. $\left.e_{6}\right)$ is incident with $v_{1}$ (resp. $\left.v_{2}\right)$ and with another vertex, which we denote by $v_{3}$ (resp. $v_{4}$ ). There are four possible ways (up to symmetry) of the (one-edge or two-edge) blow up. The one-edge blow up with the center $e_{4}$ yields two admissible indices $(9,5,2,1,1)$ and $(10,5,1,1,1)$. Both have already been found in the preceding subsections.

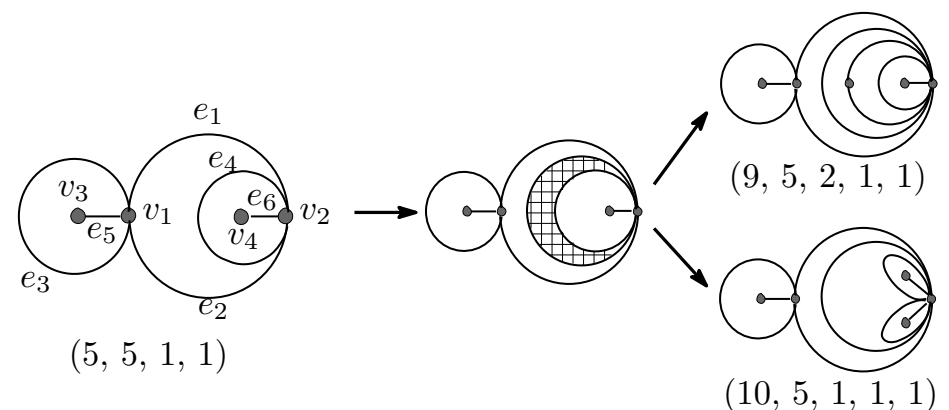

Fig. 5.15

Taking the one edge-blow up with the center $e_{1}$, we obtain two admissible indices $(9,6,1,1,1),(7,7,2,1,1)$. 


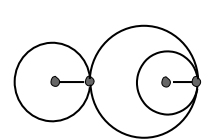

$(5,5,1,1)$

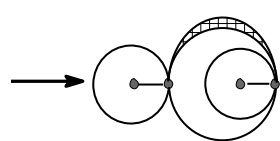

(

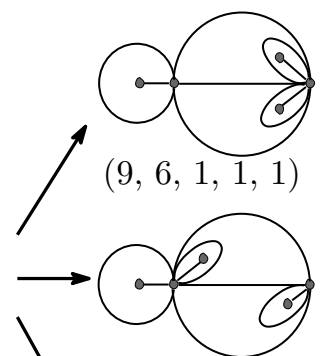

$(9,6,1,1,1)$

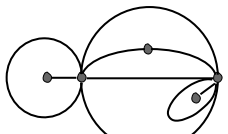

$(7,7,2,1,1)$

Fig. 5.16

By taking the one-edge blow up with the center $e_{5}$ yields three admissible indices $(9,5,2,1,1),(6,5,5,1,1),(7,5,3,2,1)$, but the first one has already been obtained.

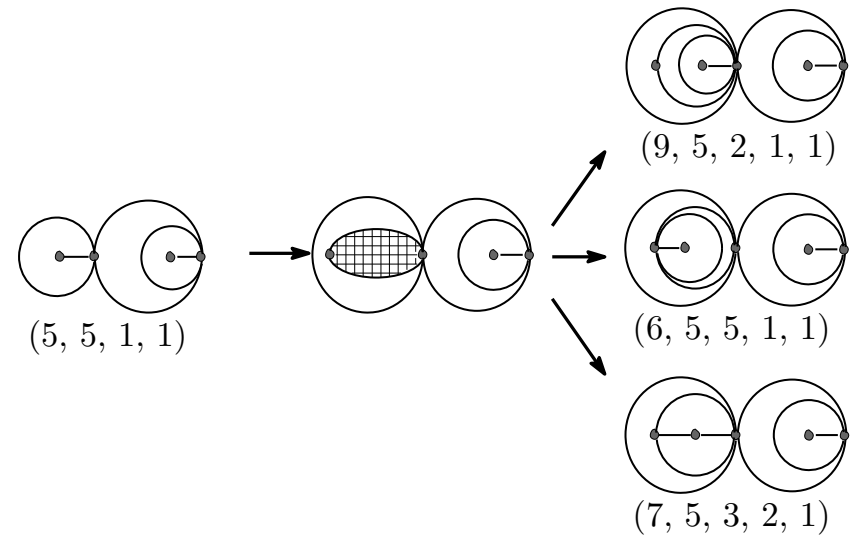

Fig. 5.17

The two-edge blow up with the centers $e_{1}, e_{2}$ yields an admissible index $(7,6$, $3,1,1)$.

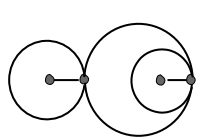

$(5,5,1,1)$

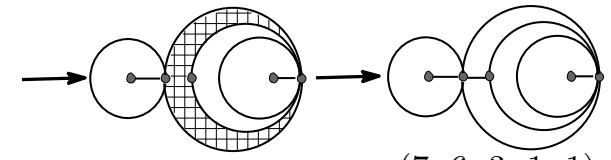

$(7,6,3,1,1)$

Fig. 5.18 
Hence we have four new admissible indices:

$$
(7,7,2,1,1),(6,5,5,1,1),(7,5,3,2,1),(7,6,3,1,1)
$$

\section{$\S 5.6$. The case of $(6,3,2,1)$}

Let $\mathcal{T}^{\prime}$ be a specific triangulation of genus one with index $(6,3,2,1)$. Let $v_{1}, v_{2}, v_{3}$ and $v_{4}$ denote vertices of $\mathcal{T}^{\prime}$ of degree $6,3,2,1$, respectively. The edge incident with $v_{1}, v_{4}$ is denoted by $e_{1}$. Let $e_{2}$ and $e_{3}$ denote edges incident with $v_{1}, v_{3}$ and $v_{3}, v_{2}$, respectively. There are two edges incident with $v_{1}, v_{2}$. We denote them by $e_{4}, e_{5}$. There is one more edge, which is denoted by $e_{6}$. There are eight possible ways (up to symmetry) of the one-edge or the two-edge blow up of $\mathcal{T}^{\prime}$. The one-edge blow up with the center $e_{1}$ yields admissible indices $(7,5,3,2,1),(10,3,2,2,1),(8,3,3,2,2)$. The first two have already been found in Sections 5.4 and 5.5.

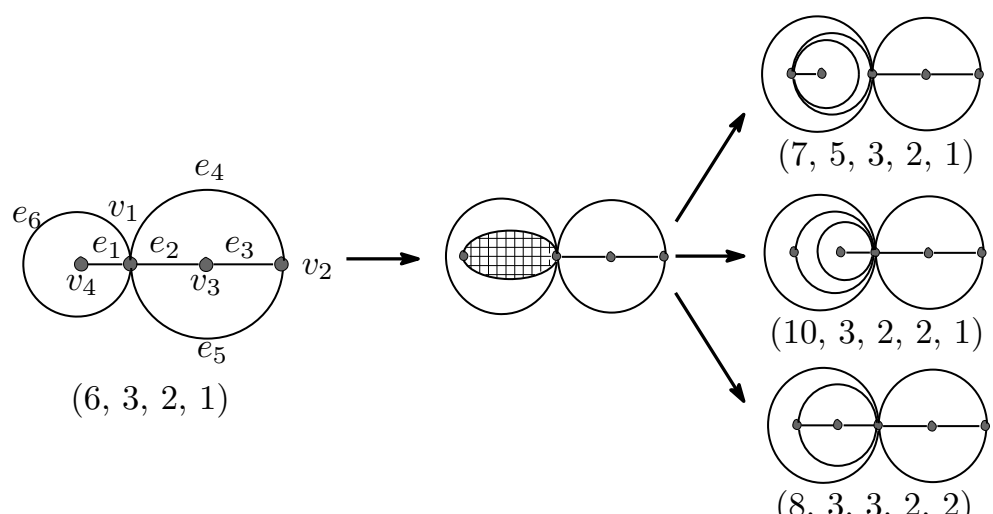

Fig. 5.19

The one-edge blow up with the center $e_{2}$ yields admissible indices $(10,3,3,1$, $1),(7,6,3,1,1),(8,4,3,2,1)$. All these indices have already been obtained in the preceding subsections. 


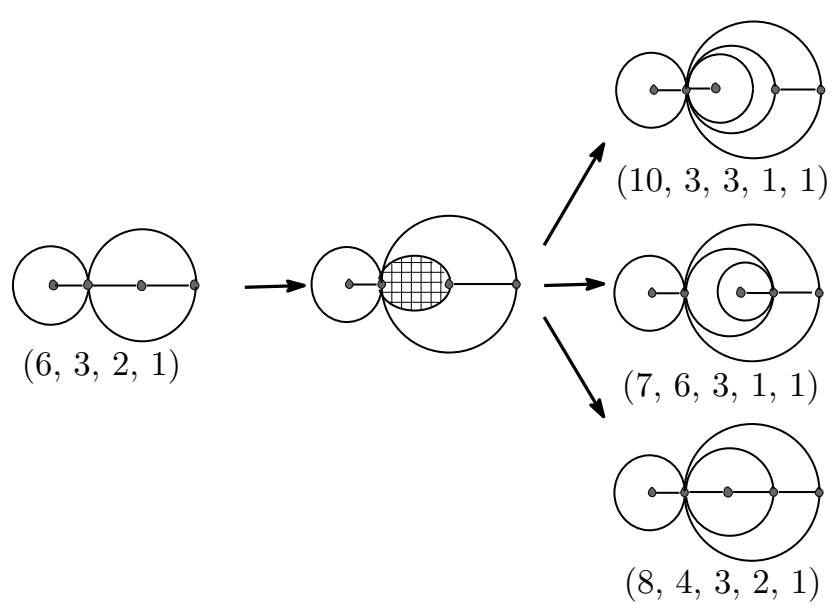

Fig. 5.20

Taking one-edge blow up with the center $e_{3}$, we have admissible indices $(6,6$, $4,1,1),(7,6,3,1,1),(6,5,4,2,1)$. The first one is new.

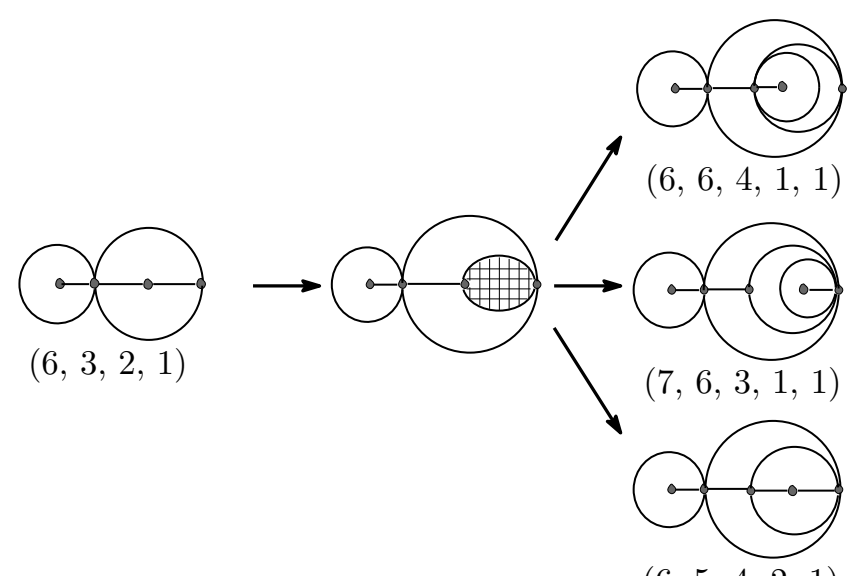

Fig. 5.21

The one-edge blow up with the center $e_{4}$ yields admissible indices $(10,4,2,1$, $1),(7,7,2,1,1),(8,5,2,2,1)$. All these indices have already been obtained in the preceding subsections. 


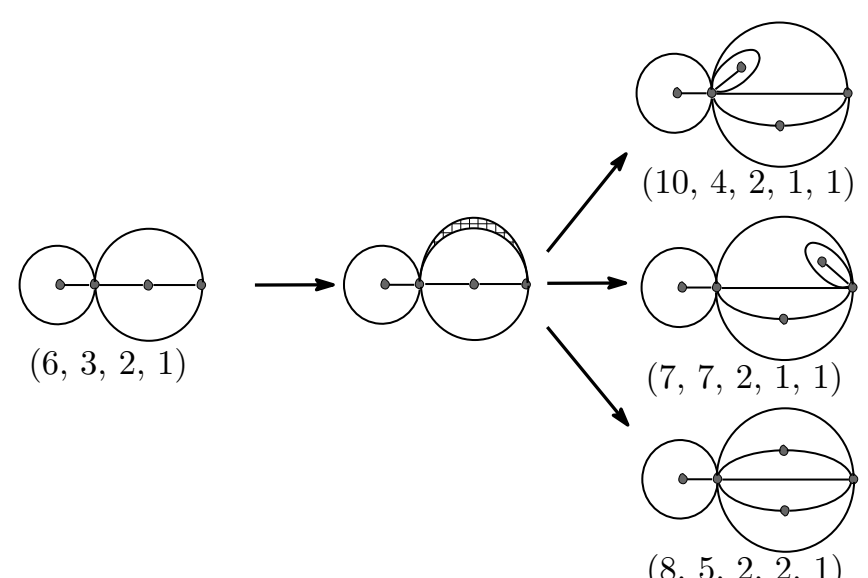

Fig 5.22

The one-edge blow up with the center $e_{6}$ yields admissible indices $(10,3,2$, $2,1),(11,3,2,1,1)$, which have already been found.

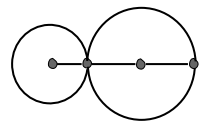

$(6,3,2,1)$
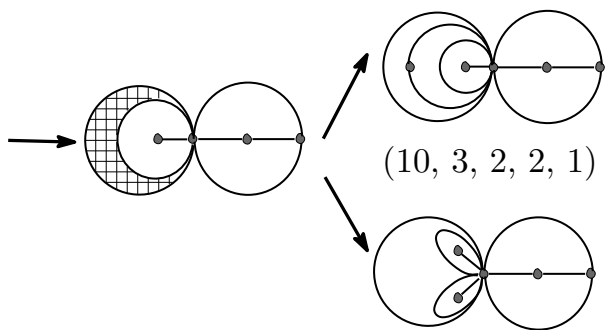

$(11,3,2,1,1)$

Fig 5.23

The two-edge blow up with the centers $e_{4}, e_{5}$ yields an admissible indices $(8,4,3,2,1)$, which has been obtained in Section 5.2.

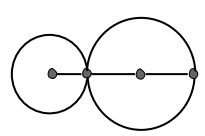

$(6,3,2,1)$

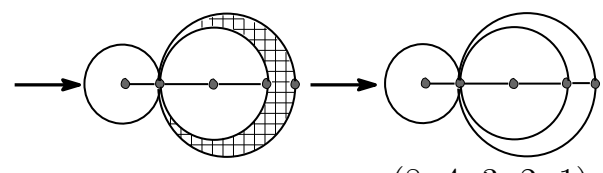

$(8,4,3,2,1)$

Fig 5.24

The two-edge blow up with the centers $e_{2}, e_{3}$ (resp. $e_{4}, e_{6}$ ) yields an admissible index $(7,4,3,3,1)$ (resp. $(8,4,3,2,1))$ which has already appeared. 


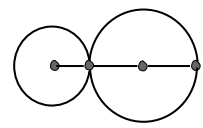

$(6,3,2,1)$

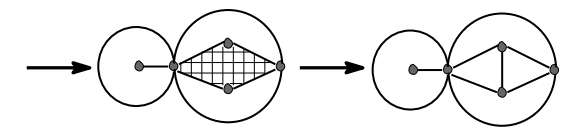

$(7,4,3,3,1)$

Fig 5.25

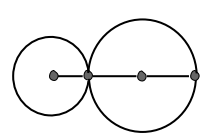

$(6,3,2,1)$

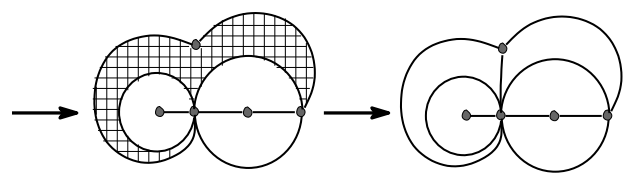

$(8,4,3,2,1)$

Fig 5.27

Hence we have obtained two new admissible indices:

$$
(8,3,3,2,2),(6,6,4,1,1) .
$$

\section{$\S 5.7$. Finish of the proof}

By the discussion in Sections 5.1-5.6, we see that 25 multi-indices in the statement of Theorem 2.1 are admissible and, at the same time, we obtain configurations of abstract specific triangulations with these indices. This completes the proof of Theorems 2.1 and 2.2 .

\section{§6. Appendix}

For each Stokes graph $\mathcal{S}$ given in Theorem 2.3, we can find potentials $Q$ so that the Stokes graphs of (1.1) coincide with $\mathcal{S}$ by using numerical experiments. We give an example of such a $Q$ for each admissible index. Here we note that, by taking suitable Möbius transformations, we consider the case where all of the regular singularities are finite. Hence the degree of $G$ in the following examples is $g+3=5$.

(i) $(4,4,4,3,3)$ :

$$
Q(x)=\frac{(x-(1-i))(x-(1-3 i))(x-2 i)(x+4 i)(x+(1+i))(x+(1+3 i))}{(x-1)^{2} x^{2}(x+2 i)^{2}(x+3 i)^{2}(x+1)^{2}} .
$$

(ii) $(5,5,3,3,2)$ :

$$
Q(x)=\frac{(x-(1-3 i)) x(x+i)(x-2 i)(x+4 i)(x+(1+3 i))}{(x-1)^{2}(x-i)^{2}(x+2 i)^{2}(x+3 i)^{2}(x+1)^{2}} .
$$


(iii) $(6,4,4,2,2)$ :

$$
Q(x)=\frac{(x-(1+i))(x-(1-i))(x-2 i)(x+2 i)(x+(1-i))(x+(1+i))}{(x-2)^{2}(x-1)^{2} x^{2}(x+1)^{2}(x+2)^{2}} .
$$

(iv) $(6,5,4,2,1)$ :

$$
Q(x)=\frac{(x-(2-i))(x-(1-i))(x+3 i)(x-4 i)(x+(1+i))(x+(2+i))}{x^{2}(x-i)^{2}(x+i)^{2}(x-2 i)^{2}(x-3 i)^{2}} .
$$

(v) $(6,5,5,1,1)$ :

$$
Q(x)=\frac{(x-i)(x+i)(x-2 i)(x+2 i)\left(x-\frac{9 i}{2}\right)\left(x+\frac{9 i}{2}\right)}{x^{2}\left(x-\frac{5 i}{2}\right)^{2}\left(x+\frac{5 i}{2}\right)^{2}(x-3 i)^{2}(x+3 i)^{2}} .
$$

(vi) $(6,6,2,2,2)$ :

$$
Q(x)=\frac{(x-i)(x+i)(x-2 i)(x+2 i)(x-4 i)(x+4 i)}{(x-2)^{2} x^{2}(x-3 i)^{2}(x+3 i)^{2}(x+2)^{2}} .
$$

(vii) $(6,6,4,1,1)$ :

$$
Q(x)=\frac{(x-(2-2 i))(x+i)(x+2 i)(x-4 i)(x+4 i)(x+(2+2 i))}{x^{2}(x-i)^{2}(x-2 i)^{2}(x-3 i)^{2}(x+3 i)^{2}} .
$$

(viii) $(7,4,3,3,1)$ :

$$
Q(x)=\frac{(x-(1-2 i)) x(x+i)(x-3 i)(x+3 i)(x+(1+2 i))}{(x-(1-i))^{2}(x-i)^{2}(x-2 i)^{2}(x+2 i)^{2}(x+(1+i))^{2}} .
$$

(ix) $(7,5,3,2,1)$ :

$$
Q(x)=\frac{(x-(1+2 i)) x(x+i)(x-4 i)(x+4 i)(x+(1-2 i))}{(x-i)^{2}\left(x+\frac{3 i}{2}\right)^{2}(x-2 i)^{2}(x+2 i)^{2}(x-3 i)^{2}} .
$$

(x) $(7,6,3,1,1)$ :

$$
Q(x)=\frac{(x-(1-3 i))\left(x-\frac{i}{2}\right)(x-i)(x-4 i)(x+4 i)(x+(1+3 i))}{x^{2}(x+i)^{2}(x-2 i)^{2}(x-3 i)^{2}(x+3 i)^{2}} .
$$

(xi) $(7,7,2,1,1)$ :

$$
Q(x) \frac{(x-(1+3 i))(x-i)(x+i)(x-4 i)(x+4 i)(x+(1+3 i))}{(x-2)^{2}\left(x-\left(\frac{3}{2}+2 i\right)\right)^{2} x^{2}\left(x+\left(\frac{3}{2}+2 i\right)\right)^{2}(x+2)^{2}} .
$$


(xii) $(8,3,3,2,2)$ :

$Q(x)=\frac{\left(x-\left(\frac{1}{2}+i\right)\right)\left(x-\left(\frac{1}{2}-i\right)\right)(x-3 i)(x+3 i)\left(x+\left(\frac{1}{2}-i\right)\right)\left(x+\left(\frac{1}{2}+i\right)\right)}{x^{2}(x-i)^{2}(x+i)^{2}(x-2 i)^{2}(x+2 i)^{2}}$.

(xiii) $(8,4,3,2,1)$ :

$Q(x)=\frac{(x-1)(x-(1-2 i))(x+3 i)(x-4 i)(x+1)(x+(1+2 i))}{(x-(1-i))^{2}(x+i)^{2}(x-2 i)^{2}(x-3 i)^{2}(x+(1+i))^{2}}$.

(xiv) $(8,5,2,2,1)$ :

$$
Q(x)=\frac{(x+i)(x-2 i)(x+2 i)(x-3 i)(x-5 i)(x+5 i)}{x^{2}(x-i)^{2}\left(x+\frac{12 i}{5}\right)^{2}\left(x+\frac{14 i}{5}\right)^{2}(x-4 i)^{2}} .
$$

(xv) $(9,5,2,1,1)$ :

$$
Q(x)=\frac{\left(x-\left(\frac{1}{2}+2 i\right)\right)(x+i)(x+2 i)(x-4 i)(x+4 i)\left(x+\left(\frac{1}{2}-2 i\right)\right)}{x^{2}(x-i)^{2}(x-2 i)^{2}(x-3 i)^{2}(x+3 i)^{2}} .
$$

(xvi) $(9,6,1,1,1)$ :

$$
Q(x)=\frac{(x-(1-3 i)) x\left(x-\frac{i}{2}\right)(x-4 i)(x+4 i)(x+(1+3 i))}{(x-i)^{2}(x+i)^{2}(x-2 i)^{2}(x-3 i)^{2}(x+3 i)^{2}} .
$$

(xvii) $(10,3,2,2,1)$ :

$$
Q(x)=\frac{(x-1)(x-(1-2 i)) x(x-4 i)(x+4 i)(x+(2-3 i))}{(x-3)^{2}(x+3 i)^{2}(x+1)^{2}(x+2)^{2}(x+(2-2 i))^{2}} .
$$

(xviii) $(10,3,3,1,1)$ :

$$
Q(x)=\frac{(x-(1-2 i))\left(x-\frac{i}{2}\right)(x-i)(x+3 i)(x-4 i)(x+(1+2 i))}{\left(x-\left(1-\frac{3 i}{2}\right)\right)^{2}(x+i)^{2}\left(x-\frac{3 i}{2}\right)^{2}\left(x-\frac{5 i}{2}\right)^{2}\left(x+\left(1+\frac{3 i}{2}\right)\right)^{2}} .
$$

(xix) $(10,4,2,1,1)$ :

$$
Q(x)=\frac{(x-i)(x+i)(x-2 i)(x+2 i)(x-4 i)(x+4 i)}{x^{2}\left(x-\frac{i}{2}\right)^{2}\left(x+\frac{i}{2}\right)^{2}(x-3 i)^{2}(x+3 i)^{2}} .
$$


(xx) $(10,5,1,1,1)$ :

$$
Q(x)=\frac{(x-1)(x-(1-2 i))(x-3 i)(x+4 i)(x+1)(x+(1+2 i))}{(x-(1+i))^{2}(x-i)^{2}(x-2 i)^{2}(x+3 i)^{2}(x+(1-i))^{2}} .
$$

(xxi) $(\mathbf{1 1}, 3,2,1,1)$ :

$Q(x)=\frac{(x-(1+2 i))(x-(1-2 i))(x+3 i)(x-4 i)(x+(1-2 i))(x+(1+2 i))}{(x-(1-i))^{2} x^{2}(x-i)^{2}(x-3 i)^{2}(x+(1+i))^{2}}$.

(xxii) $(12,2,2,1,1)$ :

$$
Q(x)=\frac{(x-i)(x+i)(x-2 i)(x+2 i)(x-4 i)(x+4 i)}{x^{2}\left(x-\frac{i}{2}\right)^{2}\left(x+\frac{i}{2}\right)^{2}(x-3 i)^{2}(x+3 i)^{2}} .
$$

(xxiii) $(\mathbf{1 2 , 3 , 1 , 1 , 1 ) : ~}$

$$
Q(x)=\frac{(x-1)(x-(1-2 i))(x-3 i)(x+4 i)(x+1)(x+(1+2 i))}{(x-(1+i))^{2}(x-i)^{2}(x-2 i)^{2}(x+3 i)^{2}(x+(1-i))^{2}} .
$$

(xxiv) $(13,2,1,1,1)$ :

$$
Q(x)=\frac{(x-(1-2 i))(x-i)(x-2 i)(x+3 i)(x-4 i)(x+(1+2 i))}{(x-(1-i))^{2} x^{2}\left(x-\frac{i}{2}\right)^{2}(x-3 i)^{2}(x+(1+i))^{2}} .
$$

(xxv) $(14,1,1,1,1)$ :

$Q(x)=\frac{(x-(1+2 i))(x-(1-2 i))(x-3 i)(x+3 i)(x+(1-2 i))(x+(1+2 i))}{(x-(1+i))^{2}(x-(1-i))^{2} x^{2}(x+(1-i))^{2}(x+(1+i))^{2}}$.

We also give the Stokes geometries for them. In the following figures, small disks designate regular singular points and larger ones turning points.

(i)

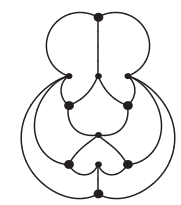

(ii)

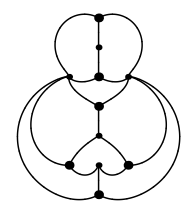

Fig. A.1

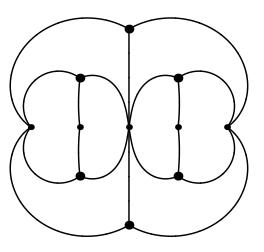

(iii) 


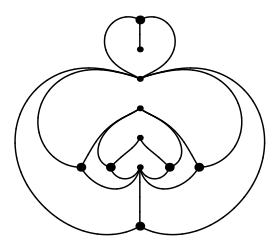

(iv)

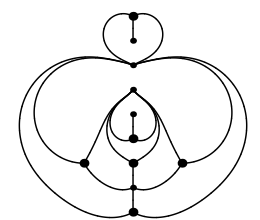

(vii)

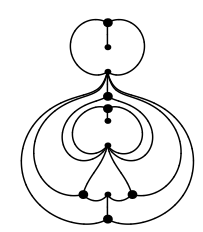

(x)

(xiii)

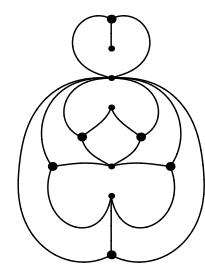

(xiv)

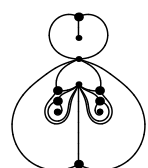

(xvi)

(v)

(viii)

(xi)

(xvii)
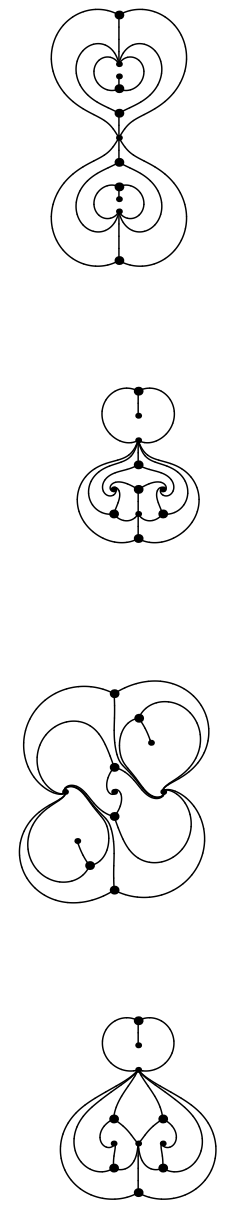

(xii)

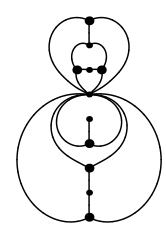

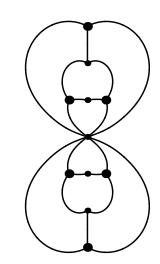

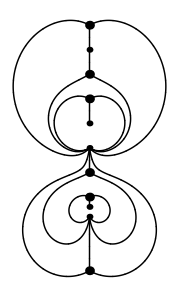

(xv)

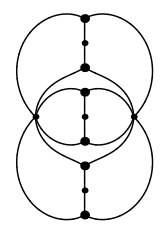

(vi)

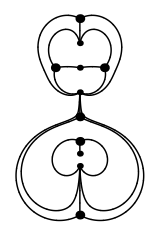

(ix)

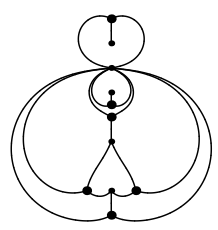

(xviii)

Fig. A.2 
(xix)

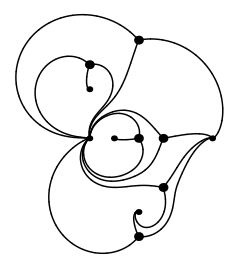

$(\mathrm{xx})$
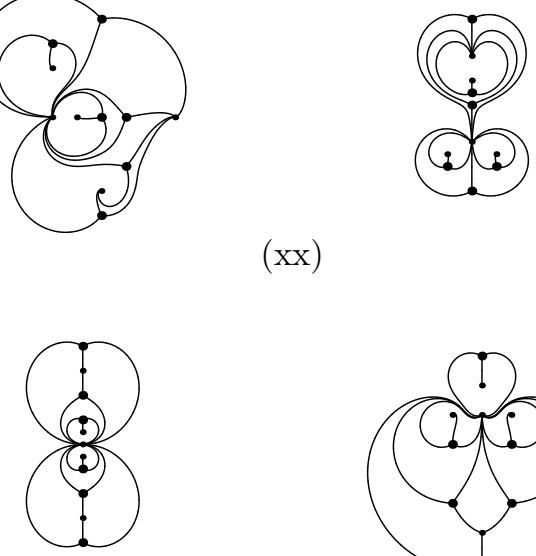

(xxii)

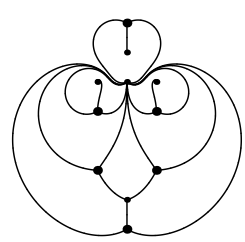

(xxiii)

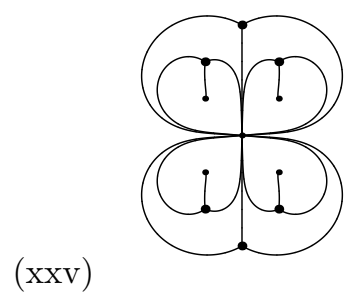

(xxv)

Fig. A.3

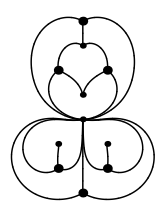

(xxi)

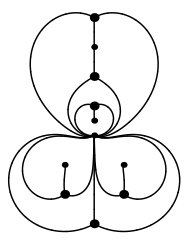

(xxiv)

These figures are drawn by using Mathemtaica. Once the Stokes geometry is obtained, one can compute the monodromy matrices for Equation (1.1) with respect to the WKB-solution basis by using the exact WKB analysis [1], [4].

\section{Acknowledgements}

The authors would like to thank Professor Shinsei Tazawa for many suggestions concerning graph theory.

\section{References}

[1] T. Aoki, T. Kawai and Y. Takei, Algebraic analysis of singular perturbations - on exact WKB analysis [translation of Sugaku 45 (1993), no. 4, 299-315], Sugaku Expositions 8 (1995), no. 2, 217-240.

[2] T. Iizuka, On the classification of Stokes graphs of genus 2 (in Japanese), Kinki University master's thesis (2002).

[3] T. Iizuka and T. Aoki, On Stokes graphs of genus two, J. School Sci. Eng. Kinki Univ., 40 (2004), 1-3. 
[4] T. Kawai and Y. Takei, Algebraic Analysis of Singular Perturbation Theory, Translation of Mathematical Monographs, vol. 227, AMS, 2005.

[5] M. Sato, T. Aoki, T. Kawai and Y. Takei, Algebraic analysis of singular perturbation, RIMS Koukyuuroku, No. 750, 1991, pp. 43-51 (Notes by A. Kaneko) (in Japanese).

[6] A. Voros, The return of the quartic oscillator: the complex WKB method, Ann. Inst. H. Poincaré Sect. A (N.S.) 39 (1983), no. 3, 211-338. 